Aus dem Medizinhistorischen Institut der Universität Zürich

(Direktor: Prof. Dr. med. Erwin H. ACKerKNeCht)

\title{
Genfer Medizinalstatistik und Hygiene in der ersten Hälfte des 19. Jahrhunderts: André-Louis Gosse (1791-1873), Jacob-Marc d'Espine (1805-1860) und Henri-Clermond Lombard (1803-1895)
}

\section{Von Eduard-Rudolf Müllener}

Ein 1883 in Genf erschienener Catalogue des publications relatives à l'hygiène par des auteurs Genevois ${ }^{1}$ zählt für die Epoche zwischen etwa 1800 und rund 1870 über 150 einschlägige Originalarbeiten auf, wobei in dieser Zahl die vielen der Pockenvakzination gewidmeten Publikationen (als ein Sondergebiet) noch nicht eingerechnet sind. Von den erwähnten 150 Veröffentlichungen stammen wiederum mehr als 60, d.h. fast die Hälfte, aus der Feder der drei Genfer Ärzte André-Louis Gosse, Jacob-Marc D'Espine und Henri-Clermond Lombard. Der Katalog von 1883 ist aber hinsichtlich der Werke dieser drei Männer noch unvollständig, und eine genauere Überprüfung ihrer Schriften ergibt, daß sie zusammen in der ersten Hälfte des 19. Jahrhunderts an die 90 der Hygiene im weitesten Sinne zugehörige Publikationen veröffentlicht haben. Zu diesem Grundstock, der hier näher untersucht werden soll, stoßen dann noch einzelne Beiträge z.B. von Antoine Baumgartiner (1808-1895), Frédéric Rilliet (1814-1861), François D'Ivernois (1757-1842), Louis Odier (1748-1817) und anderer Autoren, die hier gelegentlich Erwähnung finden werden. Es liegt also in Genf ein - zumindest quantitativer - Reichtum an hygienischen und medizinalstatistischen Beiträgen aus der ersten Hälfte des 19.Jahrhunderts vor, welcher zweifellos eine genauere Würdigung rechtfertigt. Dies um so mehr, als er in einer Stadt geschaffen wurde, die zu jener Zeit weder über eine Universität noch über einen Lehrstuhl oder ein Institut für Hygiene verfügte, die keine sich mit Hygiene oder Sozialmedizin befassende Körperschaft aufwies, deren öffentliche Gesundheit von einer behelfsmäßigen und durch die Regierung bewußt «temporär» gehaltenen «chambre de santé»

1 A.J.Duval und P.-L.Dunant, Publication de la Société d'Hygiène de Genève, $\mathrm{n}^{\circ} 1$, Genève 1883. 
(oder «conseil de santé») überwacht wurde, und die bis 1848 einem lokkeren Staatenbunde und anschließend einem Bundesstaat angehörte, der erst in der zweiten Hälfte des 19. Jahrhunderts eine eindeutige Zentralisierung des Gesundheitswesens und der Statistik zustande brachte ${ }^{2}$. Es fehlten also in Genf (und in der Schweiz überhaupt) nicht nur die wissenschaftlichen Grundlagen und Möglichkeiten der Medizinalstatistik und Hygiene, sondern (gesamtschweizerisch betrachtet) auch die nicht zu unterschätzenden politischen und sozialen Voraussetzungen für ein Gedeihen dieser Wissenschaften: der starke, zentralistisch geleitete Staat und die Industrialisierung, Umstände, die z.B. Frankreich im Zusammenhang mit den allgemeinen medizinischen Fortschritten der Pariser Medizinschule in den ersten zwei Dezennien des 19. Jahrhunderts zu seiner führenden Stellung in der öffentlichen Gesundheitspflege aufsteigen ließen und die nicht zuletzt Großbritannien in derselben Zeitepoche zu seiner Spitzenstellung auf dem Gebiet der Medizinalstatistik (Mortalitätsfragen, Todesursachen) verhalfen. Unter den ungünstigen gesamtschweizerischen Bedingungen brachte denn in unserem Lande - abgesehen von vereinzelten Beiträgen aus andern Regionen - auch nur Genf von 1800 bis etwa 1860 eine einigermaßen beachtenswerte Leistung in diesen Wissenschaften zustande. Sie ist vor

2 Die Gründung der Universität Genf fand 1872 statt. Der erste Lehrstuhlinhaber für Hygiene war von 1876 bis 1889 Pierre-Louis Dunant (1834-1918). Die "Société d'Hygiène de Genève» wurde 1883 gegründet. $\mathrm{Zu} \mathrm{«Chambre»} \mathrm{und} \mathrm{«Conseil} \mathrm{de} \mathrm{Santé»}$ vgl. folgende Protokollauszüge im Recueil des lois depuis 1812 et feuille fédérale (im Genfer Staatsarchiv): 26. Juli 1815: «Il sera formé une chambre de santé.»19.Dezember 1828: «Il sera créé un conseil de santé... supprimant la chambre de santé.» 3. Mai 1850: «Le conseil de santé est dissout.» Begründung: «Le Conseil d'Etat... considérant que le conseil de santé était un comité auxiliaire permanent contraire à la constitution...» («comités auxiliaires» dürfen nur «temporaires» sein). «Le département de justice et police est autorisé à organiser, s'il le juge convenable, un Bureau de salubrité publique, et à composer lui-même, le cas échéant, toute comission spéciale temporaire, appelée à donner des préavis à l'administration sur les questions relatives à la santé publique... » Die Gründung des Eidgenössischen Gesundheitsamtes erfolgte erst 1890, jene des Eidgenössischen Statistischen Büros 1860. Vor 1890 waren einige eidgenössische Gesundheitsgesetze erlassen worden, und der Bundesrat ließ sich gelegentlich von ärztlichen Kommissionen beraten, ebenso die Tagsatzung vor 1848. Statistische Erhebungen (Volks-, Viehzählungen u.a.m.) erfolgten in einzelnen Kantonen schon in früheren Jahrhunderten, und einige hatten auch bereits vor 1860 kantonale statistische Büros errichtet (s. H. Carrière, L'hygiène publique en Suisse, Genève 1900; J.J.KumMER, Geschichte der Statistik in der Schweiz, Zeitschrift für Schweizerische Statistik 21 [1885] 1-32). 
allem den drei praktischen Ärzten Gosse, D'Espine und Lombard zu verdanken, die sich ohne entsprechende Spezialausbildung, meist ohne offiziellen Auftrag und ohne jegliche Aussicht auf akademische Anerkennung damit beschäftigten. Diese gewissermaßen privaten, tastenden Forschungen, die zusammengenommen doch schon ein recht imponierendes Gebäude ergeben, sind die ersten schweizerischen Gehversuche auf dem Gebiet der modernen öffentlichen Gesundheitspflege.

Damit behält Genf die um rund 1800 errungene führende Stellung in der schweizerischen Medizin bis um etwa 1850 bei. Seine Ärzte des ausgehenden 18. Jahrhunderts studierten meist in Großbritannien, und zwar an der Medizinschule von Edinburg, die damals als eine der besten Ausbildungsstätten überhaupt gelten konnte (z.B. Odier, Prévost und Coindet); die Anglophilie Genfs zeigt sich auch in der Gründung der Bibliothèque Britannique, eines wissenschaftlichen Periodikums, welches ursprünglich hauptsächlich als Referatenblatt für englische Publikationen gedacht war und das während der Kontinentalsperre weiterhin angelsächsisches Gedankengut verbreitete ${ }^{3}$. Da nach der Jahrhundertwende die Mehrzahl der Genfer Ärzte die Pariser «Ecole de Médecine» absolvierte (Genf gehörte von 1798 bis 1813 als Hauptort des «Département Léman» zu Frankreich), z. B. Matthey, Senn, Rilliet sowie Gosse, D'Espine und Lombard, flossen der in mehreren Vereinigungen recht straff organisierten Genfer Ärzteschaft schon frühzeitig die neuen Ideen der revolutionären französischen Medizin $\mathrm{zu}^{4}$. Die genferische Synthese von angelsächsischem und französischem Gedankengut, also der Auffassungen und Methoden der beiden damals führenden Medizinschulen von Edinburg und Paris, brachte einige sehr überzeugende Arbeiten sowie etliche vielversprechende Ansätze in verschiedenén Richtungen hervor, die deutlich aus den gleichzeitigen Forschungen der übrigen Schweiz hervorstechen, welche damals entweder überhaupt wenig aktiv oder dann (was vor allem für die deutsche Schweiz gilt) zu stark der in Deutschland vorherrschenden romantischen Medizin mit ihren spärlichen greifbaren Resultaten verpflichtet war ${ }^{5}$. Daß die Genfer Wissen-

3 «On parle trop bien Anglais à Genève», soll Napoleon gesagt haben.

4 Zwischen 1780 und 1850 bestanden in Genf fünf verschiedene medizinische Gesellschaften, von denen zu gewissen Zeiten einige nebeneinander existierten (s. J. OLIviER, Les sociétés genevoises de médecine, Revue médicale de la Suisse Romande 57 [1937] 664-686.)

5 Zu dieser Synthese und z.B. der Rolle, welche André Matthey in der Psychiatrie spielte, s. E.-R.MüLlener, Die Entstehung des Kleptomaniebegriffs, Sudhoffs Archiv 
schaftler es häufig trotz genauer eigener Beobachtung (ein Hauptprogrammpunkt der Pariser Medizinschule) bei interessanten Ansätzen bewenden lassen mußten, hat seinen Grund nicht zuletzt darin, daß die kleine Stadt ohne Universität ihnen natürlich nicht die Möglichkeiten und das umfangreiche Patientenmaterial der Pariser Spitäler zu Verfügung stellen konnte, auf welche die neue französische «Hospital»-Medizin nach der Jahrhundertwende eigentlich zugeschnitten war ${ }^{6}$.

Die Arbeiten von Gosse, D'Espine und Lombard (sowie von Odier, Rilliet, Baumgartner und einiger anderer) auf den Gebieten der medizinischen Statistik (Bevölkerungs-, Mortalitäts- und Todesursachenstatistik) sowie der Hygiene im weitesten Sinne entstanden eindeutig unter dem Einfluß angelsächsischer und französischer Vorbilder. Die medizinische Statistik $^{7}$ zerfällt definitionsgemäß in zwei Hauptgebiete, nämlich in:

1. Das Berichtswesen, die eigentliche Medizinalstatistik, welche die zahlenmäßigen Häufigkeiten medizinisch relevanter Tatsachen festhält (z. B. allgemeine Krankheitsstatistik, Mortalität, Morbidität, Letalität, Mütterund Säuglingssterblichkeit usf.). Als ihr direkter Vorläufer kann die Bevölkerungsstatistik gelten, welche die Bevölkerungszahl und ihre Bewegungen studiert.

48 (1964) 216-239. Ferner zu den Beiträgen der Genfer Ärzte zum Meningitis-Problem: E.-R. Müllener, Six Geneva Physicians on Meningitis (1779-1843), Journal of the History of Medicine and Allied Sciences (im Druck). Weiterhin: J. Olivier und G. DE Morsier, Le Dr. Gaspard Vieusseux (Genève, 1764-1814), Revue médicale de la Suisse Romande 63 (1943) 421-440. C. CASTELLANI, Le prime descrizioni della meningite cerebrospinale epidemica, Rivista di storia della medicina 5 (1961) 244-260. H.Buess, The Contribution of Geneva Physicians to the Physiology of Development in the 19th Century, Bull. Hist. Med.21 (1947) 87-97. H.BuEss, Zur Frühgeschichte experimenteller Befruchtungsstudien, Gesnerus 2 (1945) 173-191. Сн. BaEHnI, Il y a 150 ans, le Genevois J.-P. Vaucher découvrait la fécondation chez les algues, Les musées de Genève $10(1953)$ n 10 . G. Morsier und M. Cramer, Jean-Antoine Colladon et la découverte de la loi de l'hybridation en 1821, Gesnerus 16 (1959) 113-123. N. MaNi, La découverte de l'urémie expérimentale par Jean-Louis Prévost et Jean-Baptiste Dumas, Genève 1821, Médecine et Hygiène 21 (1963) 408-409.

${ }^{6}$ Siehe E.H.Ackerknecht, Kurze Geschichte der Medizin, Stuttgart 1959, S. 117. Die «Krankenhausmedizin» löste zu Beginn des 19. Jahrhunderts die frühere «Bibliotheksund Krankenbettmedizin» ab und wurde ihrerseits nach der Mitte des Jahrhunderts von der «Labormedizin» entthront.

${ }^{7}$ Statistik $=$ (vergleichende) zahlenmäßige Erfassung, Untersuchung und Darstellung von Massenerscheinungen. Statistisch = zahlenmäßig. 
2. Die Anwendung der statistischen Methodenlehre auf medizinische Probleme (z.B. die Wirksamkeit verschiedener Behandlungsarten), d.h. die Suche nach quantitativen Gesetzmäßigkeiten bei medizinischen Massenerscheinungen.

Die Definition von Statistik als zahlenmäßige Erfassung von Massenerscheinungen zeigt bereits, warum vor 1800 zwar eine Bevölkerungsstatistik, aber keine medizinische Statistik möglich war: erst das nachrevolutionäre, industrielle Paris mit seinen großen Spitälern, erst die auf genauer Beobachtung von möglichst vielen Fällen aufgebaute «Hospital»-Medizin ließen die Krankheit und die Therapie zu Massenerscheinungen werden, während früher, zur Zeit der «Bibliotheks »- und «Krankenbett »- Medizin, die Krankheit noch - außer in Seuchenperioden - gewissermaßen ein individuelles Phänomen war. Mangels einer präziseren pathologischen Anatomie und einer damit verbundenen genau beobachteten Symptomatik stellte die Krankheit bis 1800 ein mehr oder minder autonomes Wesen dar, welches (aus heutiger Sicht) meist nach seinen Hauptsymptomen benannt wurde und im Rückblick nichts anderes ist als ein relativ lockeres Symptombündel. Daher konnte eine «Krankheit» in die andere übergehen («Transmutatio morborum») - so z.B. noch bei Pinel die verschiedenen Unterformen der riesigen Klasse der «Fieber». Erst die Pariser Schule mit ihrer auf Bichats Gewebepathologie begründeten verfeinerten pathologischen Anatomie kam zur Erkenntnis, daß Krankheiten bestimmte pathologisch-anatomische Läsionen sind, die wiederum gewisse einigermaßen konstante klinische Symptome nach sich ziehen; erst jetzt konnten amöboide Monstren wie «das Fieber » genauer untersucht und in einzelne feststehende Krankheitsentitäten mit stabilen pathologischen Befunden und sich reproduzierenden Symptomen aufgeteilt werden - und erst auf Grund dieser Erkenntnisse erschien das Auszählen von Krankheiten und Krankheitserscheinungen überhaupt sinnvoll.

Die Bevölkerungsstatistik, deren Material, die Masse der Bevölkerung, schon früher in greifbarer Form vorgelegen hatte, ging der Medizinalstatistik voraus. Ihre Entwicklung beginnt im England des 17. Jahrhunderts mit William Petty (1623-1687) und John Graunt (1620-1674) ${ }^{8}$, wird weiter befruchtet durch Theoretiker wie Pascal, Fermat und Huygens sowie JaKob Bernoulli (1712, Ars conjectandi) und setzt sich im 18. Jahr-

8 Siehe G. Rosen, A History of Public Health, New York 1958, S. 110 ff.; M. Greenwood, Medical Statistics from Graunt to Farr, Cambridge 1948. 
hundert fort, z. B. durch Johann Peter Süssmilgh (1707-1767) in Deutschland (1741) sowie Daniel Bernoulli in der Schweiz (1760) ${ }^{9}$; die Aufklärung vermehrt das humanitäre Interesse sowie die Beschäftigung mit soziologischen Fragestellungen, welche sich infolge der Bevölkerungszunahme und der Industrialisierung aufdrängen, und so kommt es schließlich, im Verein mit den obenerwähnten Fortschritten in der Krankheitslehre, zur medizinischen Statistik, die in der Form der eigentlichen Medizinalstatistik über die zahlenmäßigen Erscheinungen der Krankheiten, der Todesursachen sowie der Beziehungen zwischen Krankheit und sozialen Verhältnissen berichtet. Der Unterschied zu den «medizinischen Topographien», wie sie schon das Altertum hervorgrachte, liegt in der genauen zahlenmäßigen Erfassung eines viel größeren Materials ${ }^{10}$. Bahnbrechend auf diesem Gebiet waren die Engländer Edwin Chadwick (1800-1890; Rechtsanwalt) und William Farr (1807-1883), der Franzose Louis-René VilLermé (1782-1863) sowie der Belgier Lambert Adolphe Jacques QueteLet (1796-1874) ${ }^{11}$. Aber auch reine Kliniker wie Pinel, Bichat, CorviSART, BAYLE und LeNNEC interessierten sich für die zahlenmäßige Erfassung von Krankheiten ${ }^{12}$; am eingehendsten und in besonderer Weise befaßte sich Pierre-Charles-Alexandre Louis (1787-1872) damit. Sie alle betrieben noch eigentliche Medizinalstatistik («Berichtswesen»), wobei Lours diese auf Symptomatik und Pathologie sowie Therapie einzelner Krankheiten anwandte, nicht aber die Applikation der statistischen Methodenlehre auf medizinische Probleme mit Wahrscheinlichkeitsberechnungen. Dieses schwierigere Gebiet hatte zu jener Zeit gerade erst seine Theoretiker gefunden in CaBanis (Du degré de certitude dans la médecine, 1789), Laplace (Théorie analytique des probabilités, 1812), Poisson (Re-

9 Siehe F.Huber, Daniel Bernoulli (1700-1782) als Physiologe und Statistiker, Basel 1958.

10 Für medizinisch-statistische Versuche vor 1800 (vor allem hinsichtlich der Wirksamkeit der Pockenvakzination) s. B. MILt, Empirie und das statistisch fundierte biologisch-medizinische Denken in der Geschichte, Gesnerus 13 (1956) 1-28; G.Rosen, Problems in the Appliance of Statistical Analysis to Questions of Health 1700-1800, Bull. Hist. Med.29 (1955) 27-45.

11 Siehe E.H.Aćkerknecht, Villermé and Quetelet, Bull.Hist.Med.26 (1952) 317-329; F.H.Hankins, Quetelet as a Statistician, New York 1908. M.Delabrosse, Villermé, Paris 1939.

12 Siehe E.H.Ackerknecht, Hygiene in France 1815-1848, Bull.Hist.Med.22 (1948) 117-155. Pinel veröffentlichte z. B. Statistiken über Geisteskranke und unbehandelte Malariafälle (Рн.PInex, Nosographie philosophique, Paris 1818, S. LXXVII, und Médecine clinique, Paris 1815, S. 460, 462). 
cherches sur la probabilité du jugement, 1837) und vor allem Jules GavaRRET ${ }^{13}$ (1804-1890; Principes généraux de statistique médicale, 1840), doch blieb die ausgedehnte praktische Anwendung der Methodik, z.B. auf die therapeutische Forschung, der allerneuesten Zeit (nach dem Zweiten Weltkrieg) vorbehalten; dabei hatte schon LAPLACE die Wahrscheinlichkeitsrechnung zu diesem Zwecke in Vorschlag gebracht ${ }^{14}$.

Waren sich England und Frankreich im beginnenden 19. Jahrhundert auf dem Gebiet der medizinischen Statistik ebenbürtig, so schwang Frankreich in der ersten Hälfte des Jahrhunderts in der Hygiene eindeutig obenaus und wurde von Großbritannien erst wieder überflügelt nach dem Erlaß des «General Health Act» und der Einsetzung des «General Board of Health» im Jahre 1848. 1829 erfolgte in Paris die Gründung der Annales d'Hygiène et de Médecine Légale, zu deren Editoren u. a. Esquirol, Leuret, Marc, Orfila, Parent-Duchatelet und Villermé gehörten, wobei besonders die beiden letztgenannten mit ihren Beiträgen die Zeitung bekanntmachten. Vor allem Villermé und Quetelet verhalfen der französischen Hygiene zum Siegeszug durch ihre Werke, z.B. über den Einfluß des Hungers, die Sterblichkeit in verschiedenen Pariser Quartieren, die Gefängnisse und ihre Reformbedürftigkeit, den Einfluß des Wohlergehens und der Armut auf die Sterblichkeit, die Arbeiterstädte, die Epidemien, vor allem die Cholera, den physischen und psychischen Zustand der Arbeiter in bestimmten Fabriken usf.

Dies ist die große Bewegung, unter deren Einfluß Gosse, D’Espine, LOMBARD und einige andere ihre bescheideneren Werke schufen. Etliche lokale Faktoren haben aber zweifellos an der Entstehung und besonderen Prägung der Genfer Medizinalstatistik und Hygiene mitgewirkt. Hier wäre einmal der ausgesprochen humanitäre Grundzug zu erwähnen, der die Stadt in diesem Jahrhundert beherrschte und seinen Niederschlag fand u.a. in der Gründung eines «Dispensaire» (einer Art Poliklinik für Arme) im Jahre $1820^{15}$, der Konstituierung einer «Société d'utilité Publique» (1828), den

13 Siehe M.Aubenque, Un précurseur de la statistique médicale: Jules Gavarret, Biotypologie 19 (1958) 94-98.

14 LAPLACE: «Die Überlegenheit der günstigsten Behandlungsmethode wird sich um so mehr manifestieren, je mehr die Zahl der Kranken wächst, und die Berechnung wird uns die Wahrscheinlichkeit erkennen lassen, die ihrem Nutzen und ihrer Überlegenheit über andere entspricht» (nach Fischer-Lexikon, Medizin, Band 1, S. 328).

15 Siehe u. a. A.Machard, Notice sur la fondation du dispensaire, Centenaire du dispensaire des médecins 1820-1920, Genève 1920. 
Bemühungen von Gosse um die Beschaffung billiger Lebensmittel und Getränke für die Armen und um eine Verbesserung der Gefängnisse und der schließlich 1863 in der Begründung des Roten Kreuzes durch Henri DuNANT seinen schönsten Ausdruck erreichte. Ferner zeigten die Genfer des letzten Jahrhunderts ein auffälliges Interesse an überseeischen Gebieten und ihren Problemen, was wiederum die Entwicklung der Seuchenhygiene förderte. Und schließlich bot sich die kleine Republik Genf - im Gegensatz etwa zu einer Großstadt oder einem Großstaat - als ein relativ abgeschlossenes, organisch gewachsenes und gut überblickbares Experimentierfeld für medizinalstatistische und hygienische Untersuchungen dar, wobei sie dank der vor allem seit CaLviN recht straffen Organisation und Buchführung gleichzeitig auch reichlich historisches Vergleichsmaterial zur Verfügung stellte.

Die Beiträge der Genfer Ärzte sollen hier der Übersichtlichkeit halber nicht nach Autoren, sondern nach Sachgebieten geordnet vorgelegt werden. Einige biographische Notizen zu den Hauptpersonen seien daher vorausgeschickt:

Louis-André Gosse (1791-1873) ist ein Sohn des Apothekers HenriAlbert Gosse (1753-1816), der 1815 die Schweizerische Naturforschende Gesellschaft begründete. Louis-ANDRÉ studierte von 1811 bis 1816 in Paris, bereiste anschließend Italien, Deutschland, Holland und England und kehrte 1821 nach Genf zurück, wo er eine Praxis eröffnete und gleichzeitig die ersten Schritte in der Politik machte. Von 1826 bis 1829 hielt sich der Philhellene Gosse als Flotten-Generalkommissär des Großadmirals Lord Cochrane in Griechenland auf, wo er unter anderem eine Pestepidemie beobachtete und bekämpfte ${ }^{16} .1831 / 32$ studierte er die Cholera in Schlesien, Böhmen, Österreich und Tirol. 1841 zog er sich aus der umfangreichen Praxis auf sein Landgut bei Genf zurück und lebte fortan als Privatgelehrter und Politiker. Seine zahlreichen Schriften befassen sich mit Physiologie, Chemie, Pathologie, Therapie, Hygiene (vor allem Seuchenkunde und -bekämpfung), humanitären Problemen (billige Nahrungsmittel und Getränke für die Armen, Gefängnisreform), Anthropologie, Ethnologie, Sozial-

16 Über seine Erlebnisse in Griechenland s. E.RothPLETz, Lettres du Genevois LouisAndré Gosse à sa mère pendant son séjour en Grèce (1826-1830), Paris/Genève 1920. E. Rothpletz, Correspondance entre deux philhellènes: Le docteur Louis-André Gosse et l'Amiral Lord Thomas Cochrane (1827-1828), Paris/Genève 1919. J. Olivier, Les carnets du Dr. André Gosse, Les musées de Genève 10 (1953) nº 10. 
ökonomie und Industriefragen. Die Familie Gosse stammt ursprünglich aus Süddeutschland ${ }^{17}$.

JACOB-MARC D'Espine (1805-1860), der schon früh hauptsächlich an Mathematik und Naturwissenschaften interessiert war, studierte von 1826 bis 1833 in Paris, wo er Schüler von Chomel, Andral und Louis wurde, der eben mit der "méthode numérique» die Medizin revolutionierte. Ab 1834 praktizierte D'EsPINe in Genf, wo er sich seit 1837 in zunehmendem Maße mit statistischen Aufgaben befaßte, an den internationalen statistischen Kongressen von Brüssel (1853) und Paris (1855) ein gewichtiges Wort mitredete und schließlich 1858 seine Erkenntnisse in dem großen Werk Essai analytique et critique de statistique mortuaire comparée niederlegte; er kann als einer der Begründer der Medizinalstatistik gelten. Daneben befaßte er sich auch mit Epidemieproblemen, machte sich als Ohrenspezialist einen Namen und war - ein ausgesprochen sozial empfindender Charakter als Armen- und Gefängnisarzt tätig. Er starb an einem Magenleiden im Alter von nur 55 Jahren. Die Familie d'Espine stammt aus Savoyen ${ }^{18}$.

Henri-Clermont Lombard (1803-1895) kommt aus einer ursprünglich italienischen Familie (Lombardo), die 1589 ins Genfer Bürgerrecht aufgenommen worden war. Er begann seine Studien 1822 in Edinburg und blieb seither in ständiger Verbindung mit der englischen Medizin; eine Lungenaffektion zwang ihn zu einem Aufenthalt in Italien, wo er sich bereits mit Klimatologie zu beschäftigen begann. Schließlich beendigte er seine Studien in Paris, wo ihn besonders Andral und Louis beeindruckten, und 1829 eröffnete er seine Praxis in Genf, wobei er gleichzeitig noch viele Jahre als Arzt am «Dispensaire» sowie am Genfer Spital amtierte. Seine rund 80 wissenschaftlichen Publikationen befassen sich hauptsächlich mit Klimatologie, ferner mit Seuchenlehre und -bekämpfung sowie Medizinalstatistik ${ }^{19}$.

Der besseren Übersichtlichkeit halber sollen die Beiträge dieser drei sowie einiger anderer Genfer Ärzte zur Medizinalstatistik und Hygiene nach folgendem Schema geordnet erörtert werden:

17 A.-J.Duval, André-Louis Gosse, Genève 1873. H.Gosse, Esquisse biographique de L.-A.Gosse, Genève 1874 (mit Bibliographie). J.-C.Corndet, Henri-Albert et André Gosse, Fragment biographique, Genève 1875.

18 Siehe H.Buess, Schweizer Ärzte als Forscher, Entdecker und Erfinder, Basel 1946, S. 59/60. (J.-P.DupIN), Le $D^{r}$ Marc d'Espine, Notice biographique publiée par la Société Médicale du Canton de Genève, Genève 1860 (mit Bibliographie).

19 C. Picot, Le $\mathrm{D}^{\mathrm{r}}$ Henri-Clermont Lombard, Revue médicale de la Suisse Romande 15 (1895) 116-123. 
A. Medizinalstatistik (Bevölkerungs-, Mortalitäts- und Todesursachenstatistik) $=$ MS

B. Hygiene im weitesten Sinne:

1. Klimahygiene und Klimatologie $=\mathrm{KH}$

2. Seuchenhygiene $=\mathrm{SH}$

3. Humanitäre Hygiene (Untersuchung der Verhältnisse in Gefängnissen, Wohnungshygiene usw.) $=\mathrm{HH}$

4. Gewerbehygiene $=\mathrm{GH}$

Eine nach diesen Gesichtspunkten angeordnete tabellarische Übersicht der Arbeiten von Gosse, d'Espine, Lombard, Rilliet ${ }^{20}$, d'Ivernois und ODIER zeigt folgende quantitativen Verhältnisse:

\begin{tabular}{lrrrrrrr}
\hline & MS & KH & SH & HH & GH & Total Hygiene & Gesamttotal \\
\hline GOSSE & - & - & 8 & 8 & 2 & 18 & 18 \\
D'ESPINE & 16 & - & 8 & 2 & - & 10 & 26 \\
LOMBARD & 7 & 10 & 14 & 4 & 3 & 31 & 38 \\
RILLIET & - & - & 3 & - & 1 & 4 & 4 \\
D'IVERNOIS & 12 & - & - & - & - & - & 12 \\
ODIER & 3 & - & - & - & - & - & 3 \\
\hline
\end{tabular}

Total der entsprechenden

Arbeiten in

$\begin{array}{llllllll}\text { Genf } 1810-1860 & 38 & 10 & 33 & 14 & 6 & 63 & 101\end{array}$

Es wurden also in Genf während der ersten Hälfte des 19. Jahrhunderts rund hundert einschlägige Arbeiten produziert, wobei das Hundert überschritten wird, sofern man noch die Publikationen von Baumgartner, Heyer, Mallet, De Candolle-Boissier u.a.m. (die weiter unten jeweils noch kurz Erwähnung finden sollen) dazurechnet. Dabei sticht Gosse als Seuchen- und «Humanitär»-Hygieniker, D'EsPINE als Statistiker und LomBARD als Seuchen- und Klimahygieniker hervor. Es ist natürlich unmöglich,

20 Frédéric Rilliet (1814-1861) studierte von 1833 bis 1840 in Paris als Schüler von Louis, Biett und Guersent, verbrachte nach Studienabschluß drei weitere Jahre am "Hôpital des enfants» in Paris und kehrte dann als praktischer Arzt mit speziellem Interesse an Pädiatrie nach Genf zurück. Er publizierte neben seinem Hauptwerk Traité clinique et pratique des maladies des enfants (1843, zusammen mit BARTHEZ) an die 50 Arbeiten über meist pädiatrische Themen und Tuberkulose. Siehe A.J.Duval, Le docteur Rilliet, Genève 1861 (mit Bibliographie). 
die hundert Arbeiten ausführlich zu besprechen; da etliche von ihnen einen relativ geringen Wert aufweisen, ist dies auch unnötig, und es soll hier nur von den wichtigeren die Rede sein.

Zuerst aber noch ein kurzer Hinweis auf die Anschauungen von Lours, dessen Denken sowohl D'Espine wie Lombard geformt hatte. Die «méthode numérique» von Pierre-Charles-Alexandre Louis besteht in umfassenden und möglichst genauen Beobachtungen am Lebenden und am Toten (mit Sektion des gesamten Körpers, was in den ersten Dezennien des 19. Jahrhunderts noch durchaus nicht allgemeiner Brauch war) und anschließendem Auszählen der Befunde (die Zahl ersetzt Angaben wie « oft, selten, meist») und Aufdecken der Korrelationen, z.B. zwischen Symptomen und pathologischen Veränderungen ${ }^{21}$. Es handelt sich dabei also um eine Anwendung des statistischen Berichtswesens auf die Symptomatologie, Pathologie und Therapie. Mit dieser einfachen (und uns so selbstverständlichen) Methode erreichte Louis, dessen Bücher allerdings heute eine recht langfädige Lektüre darstellen, als guter Kliniker und mit Hilfe der seit Bichat verfeinerten pathologischen Anatomie ganz erstaunliche Resultate; so gelang es ihm u.a., den Typhus endgültig aus dem wirren Konglomerat der Pinelschen «Fieber» herauszuheben ${ }^{22}$. Zu seinen begeisterten Adepten gehörten die Nordamerikaner ${ }^{23}$ und die Genfer, unter diesen vor allem D'Espine und Lombard. Leider konnten sie die Methode in Genf kaum je zur Anwendung bringen - denn die Stadt war zu klein. Nur Paris (oder andere große Städte) mit seiner enormen Zahl von Krankenhausbetten (oder allenfalls Epidemien mit hohen Erkrankungszahlen) vermochten ein derart umfangreiches Material zu liefern, daß sich eine zahlenmäßige Aufgliederung der Symptome und Autopsiebefunde überhaupt lohnte. So wich D'Espine in Genf alsbald von der Krankheitsbeschreibung (welcher er in Paris einige Arbeiten im Stile von Louis gewidmet hatte) ab und wandte sich der Bevölkerungs- und Todesursachenstatistik zu, wo sich ein zahlenmäßig genügendes Material fand, und LoMBaRD beschritt einen ähnlichen Weg zur Klimatologie mit ihren umfangreichen Unterlagen hin.

21 P.-Ch.-A.Lours, De l'examen des malades et de la recherche des faits généraux, Mémoires de la Société Médicale d'Observation 1 (1837) 1-63.

22 P.-Сн.-A.Louis, Recherches anatomiques, pathologiques et thérapeutiques sur la maladie connue sous les noms de gastro-entérite, fièvre putride, adynamique, ataxique, typhoide etc., 2 Bände, Paris 1829.

23 Siehe W.Artelt, Louis' amerikanische Schüler und die Krise der Therapie, Sudhoffs Archiv 42 (1958) 291-301. 


\section{A. Medizinalstatistik (Bevölkerungs-, Mortalitäts- undTodesursachenstatistik)}

Nach ihrer Etablierung in Genf verlegten sich sowohl Lombard wie D'EsPINE zuerst auf die Bevölkerungsstatistik. Volkszählungen hatte es in der Schweiz schon seit dem 17.Jahrhundert gegeben (Basel 1610, Zürich 1634, 1671, 1771, Bern samt Waadt und Aargau 1764); als früheste schweizerische Aufzeichnung der Bevölkerungsbewegung kann das seit 1549 genau geführte Genfer Totenregister gelten. Daneben beschäftigten sich verschiedene Privatgelehrte mit Bevölkerungsproblemen, wie im 18. Jahrhundert z.B. die Genfer Jean-Antoine Cramer (1707-1775) und Abraham Joly (1748-1812) ${ }^{24}$, in Basel Iselin (1757/58) und die beiden Daniel BerNoulli (1760 sowie 1771 und 1780), in Zürich Waser, in Bern Muret, Loys de Chesaux (beide 1766) und Heinzmann (1794), ferner Faber, Fäsi, Füssli, Normann, Durand, Meister u.a.m.; im 19. Jahrhundert Fraschini (1827 und später), der Appenzeller Arzt Titus Tobler (1835) Christian Bernoulli (1827-30, 1841, 1843), Oser (1839), Hottinger $(1847)^{25}$ sowie die Genfer Odier, Picot ${ }^{26}$, De Candolle-Boissier ${ }^{27}$, MaLLET ${ }^{28}$, D'Ivernois ${ }^{29}$, HeYer ${ }^{30}$, Lombard und D'Espine. Genf verfügt also

24 CRAmer zog die alten Register aus von 1549 bis 1760 und verfolgte die Bevölkerungsentwicklung selber von 1760 bis 1775 . Sein Originalmanuskript wurde von D'IverNors 1834 wiederentdeckt (s. E. MaLlet, Recherches historiques et statistiques sur la population de Genève etc., Annales d'Hygiène Publique et de Médecine Légale 17 [1837] 5-172, besonders 28 und 33/34). JoLY setzte CRAMERS Arbeit fort und untersuchte die letzten dreißig Jahre des 18. sowie die ersten elf Jahre des 19. Jahrhunderts; unediertes Manuskript (s. Mallet, l.c., S. 31).

25 Nach J.J. Kummer, s. Anmerkung 2.

${ }^{26}$ J.Picot, Statistique de la Suisse, Genève 1819.

27 A.L.P.P. De Candolle-Boissier, Sur la population de Genève, Genève 1814. (Alphonse Louis Pierre Pyramus de Candolle, 1806-1893, Botaniker und Politiker).

28 E. F. Mallet, s. Anmerkung 24 (Edouard Félix Mallet, 1805-1856, Advokat, Richter, Politiker).

${ }^{29}$ D'IvernoIs publizierte zwischen 1830 und 1840 ein Dutzend bevölkerungsstatistische Arbeiten, meist in der Bibliothèque Universelle (Françors D'Ivernors 1757-1842, Genfer Staatsmann, 1782 aus Genf verbannt, kehrt 1792 zurück, geht dann nach England, Gegner Napoleons, macht sich den Engländern nützlich, wird naturalisiert und geadelt; kehrt 1814 nach Genf zurück und vertritt es zusammen mit Charles Pictet DE Rochemont auf dem Wiener Kongreß, s. L. Cramer, Genève et les traités de 1815, Correspondance diplomatique de Pictet de Rochemont et de François d'Ivernois, 2 Bände, Genf/Paris 1914).

30 Théophile HeYer (1804-1871), Rechnungslehrer an der Industrieschule, Staatsarchivar. 
sowohl über die am weitesten zurückreichenden Unterlagen als auch über eine große Tradition auf dem Gebiet der Bevölkerungsstatistik, an die LOMBard und D'Espine anknüpfen konnten. Gestützt auf die alten Quellen stellte der Mediziner Odier z.B. 1797 fest, daß die mittlere Lebensdauer in Genf vom 16. bis zum 18. Jahrhundert von 18 auf 32 Jahre zugenommen hatte $^{31}$; 1814 errechnete er für die Periode von 1761 bis 1813 eine erneute Zunahme von einigen Jahren ${ }^{32}$. HeYer und LOMBard setzten seine Untersuchungen 1834 für die Jahre 1816 bis 1830 fort und kamen für diesen Zeitabschnitt auf eine mittlere Lebensdauer von rund vierzig $\mathrm{Jahren}^{33}$. Sie stellten für Genf eine allgemein längere Lebensdauer, eine schwächere Mortalität und vor allem eine sehr viel geringere Kindersterblichkeit als für bestimmte Vergleichsorte (u.a. Paris) fest; sie führen dies auf die bei normaler Anzahl von Verehelichungen relativ sehr niedrige Geburtenzahl zurück, welche jedem Kind eine ausreichende materielle Versorgung und psychologische Betreuung sichert. Ferner weisen sie auf das Blühen von Handel, Industrie und Wohltätigkeit sowie die Bemühungen in hygienischer Hinsicht hin. Schon 1832 hatte LoMBard die Abhängigkeit der Mortalität verschiedener Altersklassen von den Jahreszeiten studiert, also das Klima mit der Sterblichkeit in Verbindung gebracht ${ }^{34}$; mit dieser Arbeit, von der im Kapitel «Klimahygiene» die Rede sein wird, begann sein Übergang von der Bevölkerungsstatistik zur Klimatologie. Nur noch einmal, im Jahre 1873, hat Lombard ein bevölkerungsstatistisches Problem behandelt, die

${ }^{31}$ L. OdIER, Appendice (zu einer Rezension über einen Artikel betreffend «Politische Arithmetik»), Bibliothèque Britannique (Science et Arts) 4 (1797) 327-330.

32 L. Odier, Registres mortuaires de Genève, Bibliothèque Britannique; (Sciences et Arts) 55 (1814) 213-231.

33 T. Heyer und H.-C.Lombard, Recherches statistiques sur la mortalité de la ville de Genève... depuis 1816 jusqu'à 1830 , faisant suite aux recherches du $\mathrm{D}^{\mathrm{r}}$ Odier, Bibliothèque Universelle des Sciences, Belles-Lettres et Arts (faisant suite à la Bibliothèque Britannique, Abt. Science et Arts) 19 (Tome 56) (1834) 337-370. MaLlet bestätigte mit geringen Abweichungen 1837 sowohl die Ergebnisse von OpIER wie von HeYER und Lombard hinsichtlich der mittleren Lebensdauer, indem er zu folgenden Resultaten gelangte:

Ende des 17.Jahrhunderts: 25 Jahre

1701-1750 32 Jahre

1751-1800 34 Jahre

1801-1813 38 Jahre

1814-1833 40 Jahre

(MALlet, s. Anmerkung 24, S. 98).

34 H.-C.Lombard, De l'influence des saisons sur la mortalité à différents âges, Genève 1832. 
Hintergründe der Entvölkerung Frankreichs ${ }^{35}$. Die «Entvölkerung» bestand allerdings lediglich darin, daß Frankreich die längste Zeitspanne zur Verdoppelung der Bevölkerungszahl aufwies (165 Jahre; Schweiz 117 Jahre, Sachsen 39 Jahre). Die Bevölkerungsdichte entsprach derjenigen der übrigen europäischen Staaten (69 Bewohner per Quadratkilometer), ebenso die Zahl der Verheiratungen, die allgemeine Mortalität sowie die Kindersterblichkeit; einzig die Geburtenziffer war kleiner (sowohl legitime wie außereheliche Geburten), und diese schwache Fruchtbarkeit ist nach Lombard der Grund für die «Entvölkerung». Er schreibt sie hauptsächlich dem Geiz zu, nennt sie ein «grand mal social» und macht Verbesserungsvorschläge, wie staatliche Unterstützung des Kinderkriegens sowie Einführung einer Junggesellensteuer.

Der eigentliche Medizinalstatistiker Genfs, D'Espine, beginnt seine Karriere auf diesem Gebiet mit einem im Auftrag des «Conseil de Santé»geschriebenen, sehr sorgfältigen und umfangreichen Bericht über die Genfer Mortalität im Jahre 1838, wobei er schon hier auf die Todesursachen eingeht, also sein späteres Hauptarbeitsgebiet betritt ${ }^{36}$. Nach einer Übersicht über die Sterblichkeit im ganzen Kanton bespricht er die Sterblichkeit nach Wohnort, Geschlecht, Jahreszeit («mois meurtriers» sind Februar, März und April!) und Lebensalter; die mittlere Lebensdauer beträgt (wie schon für die Periode von 1815 bis 1833) rund vierzig Jahre und ist größer auf dem Lande als in der Stadt, was D'Espine durch die Enge der städtischen Lebensverhältnisse zu erklären versucht. Er geht dann zur Todesursachenstatistik über und macht zuerst auf deren prinzipielle Schwierigkeiten aufmerksam; nicht die geringste darunter ist die Aufstellung einer brauchbaren Klassifikation der Todesursachen. D'Espines Wahl fiel auf folgende Methode: Er stellte sie nicht a priori nach irgendeinem nosologischen System auf, sondern verwertete die Angaben der Genfer Ärzte über die 1405 Todesfälle des Jahres 1838 aus. (Jeder Arzt in Genf mußte für die unter seiner Obhut verstorbenen Patienten die Todesursache zuhanden des «Registre mortuaire» angeben). Mit diesem empirischen Verfahren, welches allerdings großes Vertrauen in das Können seiner Kollegen voraussetzte, erhielt D'Espine zweifellos eine Klassifikation, die nicht allzusehr einer bestimmten Doktrin verhaftet war und damit in der verdünnten Luft einer

35 H.-C. Lombard, De la dépopulation en France, Lyon 1873.

${ }^{36}$ J.-M.D'Espine, Essai statistique sur la mortalité du canton de Genève, pendant l'année 1838, considérée tant en général que sous le rapport nosologique, Paris 1840. 
Theorie schwebte, sondern sich einer echten Beziehung zu den Möglichkeiten, dem wissenschaftlichen Ausbildungsstand und dem Denken der praktischen Ärzte rühmen durfte. Da die solchermaßen entstandene Klassifikation der Ausgangspunkt für seine ganze spätere Tätigkeit auf dem Gebiete der Todesursachenstatistik darstellt, sei hier etwas näher auf sie eingegangen. Sie beginnt mit Todesfällen infolge äußerer (Gewalt-) Einwirkung, wie Selbstmord, Mord und Unfällen verschiedener Art. Es folgen die «unfallähnlichen Todesfälle mit krankhaften Ursachen», die gewissermaßen den Übergang zwischen den gewöhnlichen Unfällen und den Todesfällen infolge Erkrankung bilden; sie umfassen die Klassen «mort subite, mort prompte, apoplexie cérébrale, apoplexie pulmonaire, convulsions, delirium tremens, hématémèse, hémorrhagie utérine, hernie étranglée und étranglement interne». Die Todesfälle infolge Erkrankung werden prinzipiell unterteilt in "morts par maladies aiguës» und «morts par maladies chroniques». Die akuten Krankheiten wiederum zerfallen in «maladies inflammatoires simples, telles que la pneumonie, la pleurésie, la péritonite spontanée, l'entérite, etc.», und in die «maladies inflammatoires spécifiques, parmi lesquelles... l'affection typhoide, l'hydrocéphale aiguë, le croup couenneux, la coqueluche, le rhumatisme articulaire aigu, les affections épidémiques telles que la rougeole, la scarlatine, la fièvre pernicieuse, etc. ${ }^{37}$ Die chronischen Krankheiten sind hervorgerufen 1. durch das «vice scrophuleux», 2. das "vice tuberculeux, 3. das "vice squirrheux ou cancéreux» oder 4. durch «altérations chroniques des tissus sans dégénérescence tuberculeuse ou squirrheuse», wobei diese letzte Klasse natürlich die heterogenste ist, indem sie in drei Unterklassen (chronische Veränderungen der nervösen Zentren, der Thorax- und der Abdominalorgane) alles umfaßt, was nicht in die ersten drei Klassen hineingeht, von der "paralysie des aliénés» über die «maladies du cœur» bis zur «affection chronique des ovaires ». In einem Anhang bringt D'EsPINE dann auch das von FARR für die englische Todesursachenstatistik im Jahre 1839 aufgestellte Klassifikationsschema zur Kenntnis ${ }^{38}$; dieses a priori aufgestellte System unterscheidet z.B. von vornherein epidemische und sporadische Krankheiten. Die sporadischen werden wiederum aufgeteilt in Affektionen des Nervensystems und der Sinnesorgane, der Respirations-, Zirkulations-, Verdauungs-, Fortpflanzungs-, Bewegungsorgane sowie der Haut und des Unter-

37 l.c., S. 32/44.

38 l.c., S. $111 \mathrm{ff}$. 
hautgewebes, ferner in Krankheiten mit unsicherem Sitz sowie hohes Alter und gewaltsamen Tod. Ein erster Blick auf die Systeme von D'Espine und FARR zeigt bereits ihre grundsätzliche Verschiedenheit, die in späteren Ausarbeitungen noch deutlicher zutage treten und die beiden Männer zu Kontrahenten bei verschiedenen internationalen Kongressen machen wird: D'Espines Klassierung geht ganz von der Praxis (nämlich von den primären Fragen, die sich bei jedem Todesfall erheben: Unfall ? unfallähnliches Ereignis? akute oder chronische Krankheit?) sowie der Ätiologie aus und ist deutlich auf die Möglichkeiten und die Denkweise der Genfer Ärzte, also letzten Endes auf die Pariser Medizinschule, zugeschnitten. Nicht umsonst kann D'Espine behaupten, er kenne «la tournure d'esprit et les habitudes nosologiques de ses collégues ${ }^{39}$ Gegen FarRS Klassierung nach Organsystemen verteidigt D'Espine sein im Sinne von Louis, Andral und СномеL nach pathologisch-anatomischen und ätiologischen Gesichtspunkten aufgebautes Schema ganz im Sinne dieser Schule, wenn er z.B. sagt: «La science, tout imparfaite qu'elle est encore sur la théorie des causes, nous enseigne... que les tubercules abdominaux ont beaucoup plus de con nexion avec ceux du thorax que la phthisie, par exemple, n'en a avec la pneumonie. ${ }^{40} \mathrm{Ihm}$ ist also weniger der «Sitz» der Todesursache wichtig, als ihr Platz in einem System der allgemeinen pathologischen Anatomie.

An D'Espines Untersuchungen über die Todesursachen in Genf erscheint noch bemerkenswert, daß «hydrocéphale aiguë» (unter welcher Bezeichnung noch bis 1840 sämtliche Formen von Meningitis liefen) 59 von 210 Todesfällen an "maladies aiguës spécifiques» ausmachen ${ }^{41}$ ! Gerade zwischen 1820 und 1840 wurde von der Pariser Schule erkannt, daß sich hinter dem «akuten Wasserkopf» meist eine tuberkulöse Meningitis verbarg ${ }^{42}$; dies war D'EsPine zweifellos bekannt, aber er konnte sich noch nicht entschließen, solche Fälle zu den chronischen (tuberkulösen) Erkrankungen (mit eventuellem akutem Ausgang) zu versetzen ${ }^{43}$. Erwähnenswert ist fer-

\footnotetext{
${ }^{39}$ l.c., S. 119.

40 l.c., S. 122.

41 l.c., S. 54.

42 Siehe dazu sowie zur Beteiligung der Genfer Ärzte Matthey, Senn und RiLliet an dieser Abklärung: E.-R. Müllener, Six Geneva Physicians on Meningitis (1779-1840), Journal of the History of Medicine and Allied Sciences (im Druck).

43 l.c. (s. Anmerkung 36), S. 55.
} 
ner, daß die tuberkulösen und skrofulösen Erkrankungen fast einen Viertel der Genfer Todesfälle im Jahre 1839 überhaupt ausmachen.

D'Espine setzte seine Untersuchungen bezüglich der Todesursachen in Genf nach seiner eigenen Klassifikation in den Jahren 1842, 1843, 1844 und 1845 fort, wobei er sich hier nun auch gegen FARRS prinzipielle Einteilung der Krankheiten in epidemische und sporadische aussprach, indem er einwandte, gerade die Frage, welche Affektionen sporadisch und welche epidemisch auftreten, wolle man ja mittels der Todesursachenstatistik erst zu beantworten versuchen ${ }^{44}$. FARR erwiderte darauf, ihm scheine es im Hinblick auf die Volksgesundheit wichtiger, die Krankheiten in epidemische und sporadische als wie D'EsPINE in akute und chronische einzuteilen womit sich D'EsPine einverstanden erklärte, aber nochmals betonte, man wisse zuwenig, um bereits jetzt a priori epidemische von sporadischen Affektionen abgrenzen zu können ${ }^{45}$. Im selben Werk befaßte sich D'EsPiNE - wie vor ihm schon Villermé - mit dem Einfluß des Wohlergehens und des Elends auf die Lebensdauer (das erste verringert die Mortalität und verlängert die mittlere Lebensdauer sowie -erwartung, besonders für Kinder, das Elend bewirkt das Gegenteil), und gleichzeitig setzte er die frühere Arbeit von Heyer und Lombard fort, indem er über die Lebensdauer und -erwartung in Genf in den Jahren 1838 bis 1845 berichtete ${ }^{46}$; die mittlere Lebensdauer war mit vierzig Jahren stationär geblieben, was D'EsPINE einigermaßen beunruhigte. Er versuchte dies mit einer gewissen Überfremdung Genfs zu erklären: die vierzig Jahre drückten daher nicht exakt die «force vitale » der eigentlichen Genfer Bevölkerung aus!

1853 beginnt D'Espines nur sieben Jahre währende brillante Karriere als international anerkannter Medizinalstatistiker. Es gelingt ihm, auf dem statistischen Kongreß von Brüssel 1853, die Todesursachenstatistik gegen den Widerstand vieler Kollegen, die sich überhaupt nichts davon versprechen, auf die Traktandenliste zu bringen, und er erhält zusammen mit

44 J.-M.D'Espine, Annuaire de la mortalité genevoise. Tableau gênéral des décès dans le canton de Genève pendant l'année 1842, Genève 1843 (dito für 1843, publiziert 1844). Ferner: Annuaire de la mortalité genevoise publié sur l'invitation du Conseil de Santé. Années 1844 et 1845, Genève 1846, S. VII.

45 J.-M. D'Espine, Influence de l'aisance et de la misère sur la mortalité, Paris 1847, S. $40 / 41$.

46 J.-M.D'Espine, Notice statistique sur les lois de mortalité et de survivance aux divers âges de la vie humaine, sur la vie moyenne et la vie probable d'après les 10203 décès qui ont eu lieu dans le canton de Genève pendant les 8 années de 1838 à 1845, Paris 1847. 
FARR den Auftrag, für den statistischen Kongreß von Paris 1855 eine international gültige Nomenklatur und Klassierung der Todesursachen auszuarbeiten ${ }^{47}$. Leider konnten sich die beiden Gelehrten besonders hinsichtlich der Klassierung nicht einigen, und jeder rückte 1855 mit einem eigenen Projekt auf, deren Grundzüge schon weiter oben abgehandelt wurden ${ }^{48}$. Die Hauptunterschiede zwischen dem Schema des Genfers und jenem des Engländers liegen sowohl in der medizinischen Ausbildung als auch in der Zielsetzung der zwei Männer begründet: D'Espine, der Pariser Schüler, stellt vor allem auf die pathologische Anatomie, die Ätiologie und die tägliche Praxis (in Genf) ${ }^{49}$ ab und will mit seinem System unbekannte Krankheitsursachen erforschen sowie Epidemiologie treiben. FARR dagegen übernimmt einfach das damals in England gültige nosologische Schema, exerziert es an sämtlichen Organsystemen so gut wie möglich durch ${ }^{50}$, setzt a

47 J.-M.D'Espine, Rapport au Conseil de Santé du canton de Genève et aux Sociétés médicales de la Suisse (Genève, Berne et Zurich), par le Docteur Marc d'Espine, leur délégué au Congrès statistique de Bruxelles (Genève le 12 octobre 1853). Im Besitz der "Société Médicale de Genève». Ich möchte an dieser Stelle Herrn Dr.med.André LENoIR, Sekretär der «Société Médicale de Genève», meinen besten Dank aussprechen für seine Hilfe bei der Beschaffung von Material.

48 J.-M.D'Espine, Projet de Classification des causes de mort physiologiques, accidentelles et morbides, à l'usage de la statistique mortuaire de tous les pays; préparé pour le congrès international de statistique de Paris (1855) - par le Docteur Marc d'Espine de Genève, l'un des deux rapporteurs. Im Besitz der "Société Médicale de Genève».

49 D'Espine schlug auch unermüdlich das in Genf seit 1536 übliche und in dieser relativ kleinen Stadt bewährte System der "vérificateurs de décès» vor, d.h. eine Totenschau durch einen (medizinischen) Beamten, der unabhängig vom behandelnden Arzt seine Meinung über die Todesursache festhielt.

50 Daher sieht es, oberflächlich betrachtet, unseren heutigen Klassierungen ähnlicher, erinnert aber bei näherer Prüfung in seiner dogmatischen Starrheit bedenklich an die Nosographien des 18. Jahrhunderts. D'EsPINE dagegen bietet kein geschlossenes System, das a priori jeder Möglichkeit gerecht werden soll, sondern betrachtet die Krankheiten als lebendige und sich entwickelnde Gestalten, deren Geschichte erst erforscht werden muß. Wie D'EsPiNe mit der Todesursachenstatistik, wollte auch August HIrsch mit der historisch-geographischen Pathologie in erster Linie ätiologische Forschung treiben (s. dazu E. BEcK, Die historisch-geographische Pathologie von August Hirsch, Gesnerus 18 [1961] 33-44, besonders 37/38). Auch heute noch versucht man unbekannte Krankheitsursachen mittels epidemiologischer Studien zu erforschen (z.B. auf dem Gebiet der Koronarerkrankungen). Zu FARR: “... Farr made the statistical method an instrument of practical reform ". Nach M. GreENwood, William Farr 18071883, in: The Medical Dictator and other Biographical Studies, London 1936, S.91-120, S. 115 . 
priori epidemische, sporadische und endemische Krankheiten fest und will primär direkt deren Auswirkungen auf die Volksgesundheit studieren. Hier stehen also die Anwendung des damaligen medizinischen Wissens zum Nutzen der Bevölkerung, die mögliche Prophylaxe von Epidemien unmittelbar im Vordergrund, dort dagegen die Erweiterung der ärztlichen Kenntnisse, das wissenschaftliche Interesse am Auftreten, Verlauf und dem Wesen der Krankheiten. D'Espines Klassierungsversuch ist also in erster Linie ein Forschungsinstrument, das allerdings den Zeitgenossen (mit Ausnahme der Franzosen) offensichtlich nur wenig einleuchtete. Der Pariser statistische Kongreß von 1855 einigte sich schließlich hinsichtlich des weniger umstrittenen Traktandums, der internationalen Nomenklatur der Todesursachen, und schloß bei der Klassierung eine Art Kompromiß zwischen D'EsPine und FARR, indem nun nicht von akuten und chronischen bzw. epidemischen und sporadischen Krankheiten, sondern von «maladie bien définie» und "maladie incomplètement définie» die Rede sein sollte, was die Sache nicht gerade klarer machte ${ }^{51}$. Der Wiener statistische Kongreß von 1857 (an welchem D'EsPINE nicht teilnahm) behielt dieses prinzipielle Schema (Klasse 1: totgeboren, 2: Tod infolge kongenitaler Schwäche oder Deformation, 3 : infolge Altersschwäche, 4: Gewalt, 5 : gut definierte Krankheit, 6 : schlecht definierte und unbekannte Krankheit) bei ${ }^{52}$, während derjenige von London 1860 (im Todesjahr von D'EsPine) noch mehr zu Farrs Auffassung hinübertendierte ${ }^{53}$. So fiel gleichzeitig mit D'Espines Ableben und dem Niedergang der Pariser Schule sowie der "méthode numérique» nach der Jahrhundertmitte auch sein dieser Forschungsmethode verpflichteter Klassierungsvorschlag in Vergessenheit; zweifellos ist es aber vor allem ihm zu verdanken, daß die Todesursachenstatistik überhaupt als Forschungsmittel internationale Beachtung fand und ein erster Versuch zur Koordinierung der diesbezüglichen Bestrebungen zustande kam. Die praktischen Resultate blieben allerdings einstweilen hinter seinen Erwartungen zurück, indem z.B. bis zum statistischen Kongreß von Berlin 1863

51 «Dem nächsten Congresse bleibt es vorbehalten, zu entscheiden, ob die in dem nosologischen Schema unter ,Tod durch genau specificirte Krankheiten' aufgeführten Krankheiten noch weiter in epidemische, endemische und sporadische oder in acute und chronische abzutheilen seien » (nach E. EngeL, Der internationale statistische Congreß in Berlin, Berlin 1863, S. 74).

${ }^{52}$ Compte-rendu général du congrès international de statistique dans ses séances tenues à Bruxelles 1853, Paris 1855, Vienne 1857 et Londres 1860, Berlin 1863, S. 85/86.

${ }^{53}$ l.c., S. 160/161. 
kein Staat die angenommene Klassierung ernsthaft zur Anwendung gebracht hatte ${ }^{54}$. Auch in der Schweiz ermöglichte erst das eidgenössische Zivilstandsgesetz vom 1.Januar 1876 eine das ganze Land umfassende Statistik der Todesursachen ${ }^{55}$. Dabei weigerten sich - unter Berufung auf das ärztliche Geheimnis - viele Ärzte (darunter z. B. ausgerechnet jene des Kantons Genf!), die Totenscheine auszufüllen. Hier schaffte erst die Einführung der konfidentiellen Sterbekarte zuhanden des statistischen Büros durch Guillaume und Schmid im Jahre 1891 Remedur; die beiden stellten auch eine neue Nomenklatur der Todesursachen auf, die im Gegensatz zur internationalen die Krankheiten nicht nach ihrem Sitz, sondern nach der Ätiologie klassifizierte - eine Idee, welche sich bereits im Schema von D'Espine angedeutet hatte ${ }^{56}$.

D'Espine setzte sich auch stark für die Förderung der Statistik in der Schweiz ein, die er auf föderalistischer Basis, unter Beiziehung von fachlich gut ausgewiesenen freien Mitarbeitern ausbauen wollte; vor der bloßen Einrichtung eines zentralen Büros mit einigen Beamten warnte er eindringlich und stellte ihm eine schlechte Prognose ${ }^{57}$. Ein solches wurde 1860 von den Bundesbehörden geschaffen und erlitt - zumindest in den Anfangsjahren - genau das von D'Espine vorausgesagte Schicksal ${ }^{58}$. Die Krönung von D'Espines Werk bildet zweifellos sein Louis gewidmeter Essai de statistique mortuaire comparée aus dem Jahre 1858, worin er seine Genfer Ergebnisse ausführlich mit sämtlichen erhältlichen Resultaten aus dem übrigen Europa vergleicht ${ }^{59}$; der «Essai» wurde von VilLERMÉ, welcher

54 «Es herrscht noch keine Übereinstimmung hinsichtlich der Classification der Todesursachen. Die den Congreßbeschlüssen entsprechendste ... ist ... die in Preußen eingeführt. Aber weder in England, noch in Frankreich, noch in Österreich, noch in Bayern usw. ist bis jetzt jenen Beschlüssen Rechnung getragen worden.» l.c. (s. Anmerkung 51), S. 86.

${ }^{55}$ Siehe H.Steiner, Die Entwicklung der Statistik der Todesursachen in der Schweiz, Bern 1902, S. 1.

56 l.c., S. 14.

57 J.-M.D'Espine, De l'organisation de la statistique officielle en Suisse, Journal de Genève, $\mathrm{n}^{\text {os }} 217,218$ und 219 (September 1859). J.-M.D'Espine, Lettre sur la création d'un bureau de statistique Suisse, Journal de Genève vom 18. Januar 1860.

58 Siehe J. J. Kummer, Geschichte der Statistik in der Schweiz, Zeitschrift für Schweizerische Statistik 21 (1885) 1-32.

59 J.-M.D'EspINe, Essai analytique et critique de statistique mortuaire comparée renfermant les monographies étiologiques des accidents et de la plupart des maladies mortelles et expliquant les lois générales de la mortalité des peuples, par les influences combinées des diverses causes de mort, Paris/Genève/Neuchâtel 1858. 
D'Espine als «une des espérances de la science» bezeichnete, sehr gelobt und zum Anlaß für die Anregung weiterer vergleichender Arbeiten auf diesem Gebiet genommen ${ }^{60}$.

\section{B. Hygiene im weitesten Sinne}

\section{Klimatologie}

Der Genfer Vertreter dieses Gebiets, Lombard, begann seine klimatologischen Forschungen 1832 mit einer Arbeit über den Einfluß der Jahreszeiten auf die Mortalität verschiedener Altersklassen ${ }^{61}$. Gestützt auf 17623 Todesfälle aus den Jahren 1779-1790 und 1816-1827, deren jahreszeitliche Verteilung er in acht verschiedenen Altersstufen (Konzeption bis Geburt, Geburt bis 1 Monat, 1 Monat bis 2 Jahre, 2-15, 15-60, 60-70, 70-80 Jahre, über $80 \mathrm{Jahre}$ ) untersuchte, kommt er zum Schluß, daß im allgemeinen der Winter mit der höchsten Mortalität verbunden ist und besonders den Neugeborenen und Kleinkindern sowie den Greisen gefährlich wird; ähnliche Feststellungen hatten vor ihm schon Villermé, Milne-Edwards und Quetelet gemacht. Lombard erklärt diese Tatsachen sehr einfach aus einer in der frühen Kindheit und im Greisenalter schwachen «force vitale » oder «résistence» und spricht sich auf Grund seiner Ergebnisse gegen die damals offenbar weitverbreitete Unsitte aus, die Neugeborenen der Kälte auszusetzen. 1833 schreibt er über das Klima von Genf und der wichtigsten Orte am Genfersee, wobei er für Personen mit «poitrine délicate» besonders die Gegend von Montreux empfiehlt ${ }^{62}$. 1856 erscheint dann seine erste größere klimatologische Arbeit, Des climats de montagne ${ }^{63}$, worin er eigene Erfahrungen und entsprechende Angaben verschiedener Freunde (die er

${ }^{60}$ L.-R. Villermé, Rappori verbal fait à l'Académie des Sciences Morales et Politiques sur un ouvrage de M. le docteur Marc d'Espine, Compte rendu des séances et travaux de l'Académie des Sciences Morales et Politiques, Cahier de Janvier 1859. Auch Quetelet empfahl die vergleichende Statistik zwischen verschiedenen Ländern eindringlich (siehe seine Rede «Sur la statistique comparative des différents pays» am Londoner Kongreß von 1860, l.c. [s. Anmerkung 52], S. 37-39).

61 H.-C. Lombard, De l'influence des saisons sur la mortalité à différents âges, Genève 1832.

${ }^{62}$ H.-C.Lombard, Du climat de Genève comparé avec celui de quelques autres localités situées au bord du lac de Genève, Bibliothèque Universelle 18 (Tome 52) (1833) 1-23, besonders 10 .

${ }^{63}$ H.-C.Lombard, Des climats de montagne considérés au point de vue médical, Genève 1856 (Neuauflagen 1858, 1873). 
einleitend erwähnt, u.a. Hermann Lebert ${ }^{64}$, dem die Publikation gewidmet ist) verwertet und das Bergklima vom meteorologischen, geographischen, physiologischen und pathologischen Standpunkt her zu umreißen versucht. Er findet eine günstige therapeutische Wirkung des Bergklimas u.a. auf Verdauungsstörungen und Appetitlosigkeit, allgemeine Schwächezustände (Rekonvaleszenz), Anämien, Hysterie und Hypochondrie, Menstruationsstörungen, Kinderkrankheiten sowie Tuberkulose; diese ist weniger häufig in Höhen von über $1000 \mathrm{~m}$ ü. M. Fortgeschrittene Fälle verlaufen allerdings in der Höhe rascher als in der Ebene, und das Höhenklima ist nur bei Abwesenheit von hektischem Fieber und wenig fortgeschrittener Tuberkulose empfehlenswert ${ }^{65}$. 1864 beschreibt er die Höhenkurorte und Bäder in den Alpen und Pyrenäen ${ }^{66}$, und in einer Arbeit aus demselben Jahr findet sich ein Hinweis darauf, daß Lombard zusammen mit einigen andern Ärzten sowie unter Mithilfe der Praktiker den Einfluß des Höhenklimas auf die Tuberkulose eingehend studieren will ${ }^{67}$. Die mehr als fünfjährige Arbeit der Tuberkulosenkommission der Schweizerischen Naturforschenden Gesellschaft fand schließlich ihren Niederschlag in einem 83 seitigen Bericht aus dem Jahre 1876, welcher erstmals über das Vorkom-

${ }^{64}$ Hermann Lebert (1813-1878), von Berlin, studierte und promovierte in Zürich, war Badearzt in Lavey (Vaud), von wo aus er auch mit Lombard in Kontakt trat; den Winter verbrachte er meist in Paris und war Mitglied der "Société Médicale d'Observation ». 1853-1859 Direktor der Medizinischen Klinik in Zürich, nachher in Breslau.

65 l.c., S. 48. Die Höhentherapie der Tuberkulose wurde von Luzius Ruedi (1804-1869), Arzt in Davos von 1835 bis 1850, im Jahre 1841 mit einer Höhenkuranstalt für skrofulöse Kinder inauguriert, nachdem er seit Jahren die merkwürdigsten Heilungen aller Grade der Skrofulose durch einen Aufenthalt in Davos beochtet hatte; ein erster diesbezüglicher Bericht aus der Feder des Zürcher Arztes Conrad Meyer-Ahrens (1813 bis 1872), der 1854 eine Monographie über die Bergkrankheit veröffentlichte, stammt von 1845. Alexander Spengler (1827-1901), der Davos zu seiner späteren Spitzenstellung als Höhenkurort für Tuberkulose verhalf, kam 1853 in das Dorf; MEYERAhrens berichtete 1862 erstmals über seine Erfolge. Herrmann Brehmer (1826-1889) begann seine Höhenlufttherapie und Sanatoriumsbehandlung der Tuberkulose in Görbersdorf 1857 (siehe dazu: G.A. WeHrLI, Zur Geschichte der Klimatotherapie, Verhandlungen der klimatologischen Tagung in Davos 1925, sowie G.A. WeHrLI, Der erste Entdecker des Davoser Höhenklimas: Dr. Luzius Ruedi, Schweizerische Medizinische Wochenschrift 7 [1926] 125-129).

${ }^{66}$ H.-C.Lombard, Les stations médicales des Pyrénées et des Alpes comparées entre elles, Genève 1864 .

67 H.-C.Lombard, Les habitants des altitudes. Leur santé et leurs maladies, Bibliothèque Universelle 21 (1864) 113-129, besonders 128/129. 
men der Tuberkulose in der Schweiz orientiert. Allerdings zeigte sich dabei der Einfluß des sozialen Standes auf die Tuberkulosehäufigkeit deutlicher als jener der Höhe über Meer: «Eines der durchschlagendsten Momente für die größere oder geringere Häufigkeit der Lungenschwindsucht, das konstant durch die ganze Untersuchung zutage trat, ist der soziale Stand, der Gegensatz zwischen agrikolen und industriellen Verhältnissen; in jenen ist die Lungenschwindsucht selten, in diesen häufig. Ebenso fest steht die Tatsache, daß mit zunehmender Höhe die Lungenschwindsucht seltener wird, obgleich dieses Moment weder so regelmäßig noch vielfach so durchschlagend sich zeigt, wie gewöhnlich erwartet und andererwärts hervorgehoben wird. ${ }^{68}$

In drei weiteren Arbeiten kehrt LoMbard wieder zu seinem Ausgangspunkt zurück, dem Einfluß der Jahreszeiten auf die Mortalität, wobei nun auch «tellurische» Gesichtspunkte vermehrt Beachtung finden; neben den einfachen Satz «le froid augmente la mortalité, tandis que la chaleur exerce une influence favorable au maintien de la vie» ${ }^{69}$ ("prédominance des décès de l'hiver et du printemps sur ceux de l'été et de l'automne») ${ }^{70}$ tritt nun die Einschränkung: «l'influence tellurique ou paludéenne diminue la force de résistence aux actions délétères qui amènent la maladie et la mort». ${ }^{71}$ Auch beg:nnt Lombard nun ethnische Gesichtspunkte vermehrt zu berücksichtigen. Auf Grund dieser Erweiterung seines Gesichtsfeldes und seiner bereits geleisteten Vorarbeiten entsteht dann bis 1877 sein monumentales Werk Traité de climatologie médicale ${ }^{72}$, das ANDRAL und Lours sowie Sir James Clark und Quetelet gewidmet ist; Lombard versucht darin eine umfassende Bearbeitung der Krankheiten nach geographischen,

68 E.MüLler, Die Verbreitung der Lungenschwindsucht in der Schweiz, Bericht der von der Schweizerischen Naturforschenden Gesellschaft zu Untersuchungen darüber niedergesetzten Kommission, erstattet von ihrem Aktuar EMIL MüLLER, Winterthur 1876, S. 74. Die Gesamtkosten der Untersuchung beliefen sich auf 620 Franken! (l.c., S.7).

${ }^{69}$ H.-C. Lombard, Des lois de la mortalité en Europe dans leurs rapports avec les influences athmosphériques, Paris 1868, S. 20.

70 H.-C.Lombard, Répartition mensuelle des décès dans quelques cantons de la Suisse, Zeitschrift für Schweizerische Statistik 4 (1868) 23-34, besonders 34.

71 H.-C.Lombard, Des influences athmosphériques sur la répartition de la mortalité à différents âges et en différents pays, Zeitschrift für Schweizerische Statistik 3 (1867) 93-111, besonders 111 .

72 H.-C.Lombard, Traité de climatologie médicale comprenant la météorologie médicale et l'étude des influences physiologiques, pathologiques, prophylactiques et thérapeutiques du climat sur la santé, 4 Bände und 1 Atlas, Paris 1877-1880. 
ethnologischen, anthropologischen, demographischen, physiologischen und pathologischen Gesichtspunkten zu geben. Methodisch fühlt er sich abdei nach wie vor Louis verpflichtet ${ }^{73}$. Ein erster Band enthält sämtliche von LOMBARD als für die Medizin relevant betrachteten meteorologischen Daten; anschließend bespricht er deren physiologische und pathologische Einflüsse auf den menschlichen Körper. Auf diese Vorarbeiten folgt im zweiten und dritten Band ein «tour du monde pathologique» von grandiosem Ausmaß, der durch einen Atlas mit 25 Karten über die geographische Verteilung der Mortalität in verschiedenen Jahreszeiten, in verschiedenen Altersstufen sowie die Verbreitung der Malaria, der Dysenterie, der Cholera, des Gelbfiebers, des Kropfs, der Lepra, der Pest u.a.m. in und außerhalb Europas illustriert wird. Ein vierter Band weist schließlich die prophylaktischen und therapeutischen Wirkungen der verschiedenen Klimata auf, wobei ethnologische und soziologische Momente nicht zu kurz kommen. "Ce sujet est l'une des plus précieuses conquêtes des temps modernes», schreibt Lombard im Hinblick auf die Klimaprophylaxe und -therapie. Damit hat er zumindest für die Tuberkulosebehandlung recht behalten, während im übrigen bald nach Erscheinen seines Werkes die Geschichte, Geographie und Klimatologie der Krankheiten von den Erkenntnissen der bakteriologischen Ära völlig in den Hintergrund verdrängt wurden. Damit fand die lange Reihe jener Arbeiten, welche Krankheiten aus dem Klima zu erklären versuchten und die sich von der Hippokratischen Schrift Über Lüfte, Wasser und Orte bis weit ins 19. Jahrhundert hinein erstreckt (z.B. Finke 1792-95, Schnurrer 1813, Boudin 1843, Fughs 1853, Mühry 1856, Hirsch 1860, Bordier 1884, Poincaré 1884, u. a.m.) ihren Abschluß. Lombards Werk läßt sich hinsichtlich Umfang und Reichhaltigkeit nicht mit der klassischen historisch-geographischen Pathologie von August Hirsch vergleichen ${ }^{74}$; es unterscheidet sich von dieser hauptsächlich darin, daß es weniger den historischen als den geographischklimatischen Gesichtspunkten verpflichtet ist, also eher eine Momentaufnahme der Weltgesundheit in den fünfziger und sechziger Jahren des letzten Jahrhunderts in ihrer Abhängigkeit vom Klima darstellt denn einen Film ihres Werdegangs. Daher ist es für den Historiker als Nachschlagewerk weniger wertvoll als Hinschs Arbeit. Dafür tritt darin um so unverfälsch-

\footnotetext{
${ }^{73}$ l.c., Band 1, S. IX: «Avec Louis nous apprîmes à connaître la valeur d'une observation minutieuse qui ne néglige aucun détail et traduit toutes ses notions par la méthode numérique.»

${ }^{74}$ Siehe dazu E.BEcK (Anmerkung 50).
} 
ter das Anliegen zutage, welches den damaligen Ärzten auf den Nägeln brannte: die Frage nach der Ätiologie und damit der Prophylaxe und Therapie all der neuen Krankheiten, die im 19.Jahrhundert erstmals erkannt oder näher umrissen worden waren. LOMBARD wendet zu diesem Zweck die Methode von LouIs (scharf beobachten und auszählen), die vieles zur Herausarbeitung neuer Krankheitsbilder beigetragen hatte, auf eine altehrwürdige Idee an, die Verursachung der Krankheiten durch das Klima. Die richtige Lösung vieler Probleme, die ihm (und andern) versagt blieb, wird ebenfalls seiner sehr alten Tradition entspringen - dem Gedanken vom Contagium vivum, der aber im Gegensatz zur Klimatheorie gerade zu Lombards Zeit von der Mehrzahl der ernsthaften und tonangebenden Forscher als endgültig überholt betrachtet wurde ${ }^{75}$.

Die Frage nach der Ätiologie der Krankheiten sowie nach der Prophylaxe und Therapie, die Lombard mit seinen klimatologischen Untersuchungen zu beantworten versuchte, unterscheidet ihn (und z.B. auch D'Espine, der ähnliches mit der Todesursachenstatistik erhellen wollte) vom Lehrmeister Louis, der sich kaum je mit diesen Problemen befaßte, sondern sich meist im Stile eines Naturwissenschaftlers auf die Erforschung der Symptome und der pathologischen Befunde beschränkte und damit neue Kranheitseinheiten schuf oder altbekannte präzisierte ${ }^{76}$. In diesem Hinaustragen der «méthode numérique» in die praktischen Bereiche der Ätiologie, Prophylaxe und Therapie besteht nicht zuletzt das Verdienst der Kleinstadtärzte unter seinen Schülern. Die Ausweitung auf praktische Belange tritt besonders bei der Seuchenhygiene hervor, da die Ärzte in den kleinen Städten Gelegenheit hatten, in übersichtlichen Verhältnissen sowohl Beginn wie Verlauf von Epidemien zu studieren (in einer Art «field trials»), während ihre Pariser Kollegen zwar eine viel größere Zahl von Fällen zu sehen erhielten, das jeweilige Krankheitsbild also eingehender erforschen,

75 Siehe E. H. Ackerknecht, Anticontagionism between 1821 and 1867, Bull. Hist. Med. 22 (1948) 562-593.

${ }^{76}$ Siehe dazu W.ARtLet, Louis' amerikanische Schüler und die Krise der Therapie, Sudhoffs Archiv 42 (1958) 291-301, besonders 293. Die Phänomene der Krankheit sind die Hauptsache, nicht die Therapie oder die Ätiologie. In Louıs' Untersuchungen z. B. über das Gelbfieber (P.-CH.-A.Louis, Recherches sur la fièvre jaune de Gibraltar en 1828, Mémoires de la Société Médicale d'Observation 2 [1844] 1-299) geht es - wie in praktisch allen Arbeiten von LouIs - ausschließlich um Symptome und Leichenbefunde, von der Verursachung (Ansteckung oder nicht?) sowie der Behandlung ist nie die Rede. 
dafür aber das eigentliche Gesicht der Epidemie weniger deutlich wahrnehmen konnten.

Die Apotheose von Lombards klimatologischem Schaffen bildet zweifellos seine Wahl zum Präsidenten des 4. internationalen Kongresses für Hygiene und Demographie im Jahre 1882 in Genf, den er mit einer Ansprache eröffnete ${ }^{77}$ und in dessen Verlaufe er selber neuerdings über die hygienischen und prophylaktischen Wirkungen des Höhenklimas redete ${ }^{78}$. Gleich anschließend sprach der damals wohl prominenteste Vertreter der Höhenphysiologie, Paul Bert. Die von Lombard stark geförderte «geographische Medizin» verlor später mit dem Beginn der bakteriologischen Ära viel Boden und lebte erst im Zusammenhang mit dem Zweiten Weltkrieg wieder auf ${ }^{79}$.

\section{Seuchenhygiene}

Die Cholera-Pandemien des 19.Jahrhunderts (1826-1837, 1840-1862, 1863-1875, und 1883-1894) erreichten auch einige Orte in der Schweiz (Lugano und Mendrisio während der ersten Pandemie; 1854 Magadino, Cadenazzo sowie Aarau und Zürich; 1855 Basel, Genf und Zürich) ${ }^{80}$. Ferner wurde Europa berührt von der Pest, welche zu Beginn des 19. Jahrhunderts in Ägypten, Syrien und der Türkei ansässig war und gelegentlich auf Nachbarländer wie Griechenland (1826-29) übergriff, sowie vom Gelbfieber, das von Zentralamerika hauptsächlich nach Spanien und Portugal importiert wurde. Mit Abflauen der Pest traten zwei auch in Europa endemische Krankheiten deutlicher hervor, der Unterleibstyphus und das Fleckfieber (Typhus z.B. in Genf 1849). Einige Erfahrungen konnten die Genfer Hygieniker also vor allem hinsichtlich der Cholera und der Typhus-Fleckfieber-Gruppe sammeln; Gosse beschrieb ferner die Pest in Griechenland. Die erste Hälfte des 19. Jahrhunderts sah mannigfache Fortschritte in der

77 P.-L.Dunant, $4^{\mathrm{e}}$ congrès international d'hygiène et de démographie à Genève (du 4 au 9 septembre 1882), Band 1 (1883) S. 96-102 (Discours d'ouverture de M. le docteux H.-C.Lombard, président du comité d'organisation).

78 H.-C.Lombard, Influences hygiéniques, prophylactiques et thérapeutiques des altitudes, l.c., Band 1 (1883) S. 180-193.

79 Siehe E.H. ACKenKNECht, Geschichte und Geographie der wichtigsten Krankheiten, Stuttgart 1963, S. $1 / 2$.

80 Nach B.Mrtw, Die Zürcher Choleraepidemie vom Jahr 1855 und die Erlebnisse eines Assistenzarztes am Kantonsspital, Vierteljahresschrift der Naturforschenden Gesellschaft in Zürich 94 (1949) 45-59, besonders 45. 
Epidemiologie und pathologischen Anatomie der erwähnten Krankheiten; im Dunkel blieb nach wie vor ihre Ätiologie, deren Abklärung erst die bakteriologische Ära zu leisten vermochte. Die alte Theorie von der Kontagion (Parasiten, Animalcula, «Contagium vivum»), die z. B. für Syphilis und Pocken schon lange angenommen worden war, galt für die Seuchen des vergangenen Jahrhunderts bis etwa 1850 sogar als überholt, und im Vordergrund stand eine Kombination von Miasma-Theorie (chemische oder physikalische Agenzien, die aus Schmutz entstehen) und soziologischhygienischen Auffassungen (Villermé, Virchow; Ursache ist der elende Zustand der Massen), wobei die Kontagion abgelehnt wurde. Wiewohl zu den beobachteten Tatsachen weder der reine Kontagionismus noch der radikale Antikontagionismus paßten, war dies jedenfalls die von den führenden medizinischen Zentren (z.B. Paris) verbreitete These - in Großstädten war eben der Ansteckungsweg schwierig zu verfolgen und die von den Antikontagionisten energisch geförderte Bekämpfung des Schmutzes und des Elends oft genug erfolgreich. In kleinen Städten lagen die Dinge anders - aber die Beobachtungen von Kleinstadtärzten hatten auch weniger Gewicht.

Die Genfer Hygieniker, welche rund 30 Arbeiten zu Seuchenfragen geliefert haben, gehörten zu den «Provinzärzten» dieser Art; ihre Beiträge zu Pest und Cholera sind allerdings eher von bescheidenem wissenschaftlichem Wert, während D'Espine und LoMbard hinsichtlich der Differentialdiagnose von Unterleibstyphus und Fleckfieber (die bis rund 1850 allgemein noch unterblieb) einige interessante frühe Bemerkungen gemacht haben, die sie leider wegen des mangelnden Patientenmaterials nicht mehr weiter verfolgen und vertiefen konnten. Die Pest und ihre Wanderungen von einer griechischen Insel zur andern im Jahre 1827 beschreibt 1838 sehr anschaulich der Griechenlandfahrer Gosse ${ }^{81}$, der den «causes prédisposantes », wie Armut, Angst, Kummer, Bevölkerungsanhäufung, Schmutz und Klimafaktoren, nur wenig Einfluß beimißt, dagegen als «cause déterminante» ein spezifisches kontagiöses Prinzip annimmt. Er unterscheidet eine «contagion immédiate», die bei der Beulenpest auftritt, indem das «virus» direkt von der Haut des einen Kranken auf jene des andern übergeht, und eine «contagion médiate», bei der das Agens bei der Lungenpest

81 L.-A.Gosse, Relation de la peste qui a régné en Grèce en 1827 et 1828, contenant des vues nouvelles sur la marche et le traitement de cette maladie, Paris 1838. 
kämpfung der Ausbreitung soll durch Quarantänen erfolgen, während die bereits Erkrankten in speziellen Lazaretten (Gosse gibt einen detaillierten Plan eines solchen) abgesondert werden müssen. Bemerkenswert ist, daß Gosse diese Ansichten noch 1838 vertritt, nachdem 1825 hauptsächlich unter dem Einfluß von MacLean die Pest-Quarantäne in England gelokkert und 1841 in Österreich und England völlig aufgehoben wurde, also eine deutliche antikontagionistische Strömung vorherrschte ${ }^{83}$. Schon in einem (weiter nicht interessanten) Buch über Rheumatismus (1826) hatte durch die Luft oder mit dem Atem von Mensch zu Mensch fliegt ${ }^{82}$. Die Besich Gosse für die "generatio spontanea» ausgesprochen ${ }^{84}$, was damals gang und gäbe war, und von «épidémies contagieuses » gesprochen ${ }^{85}$, woran er auch in einer Zeit des steigenden Antikontagionismus festhielt.

Die Cholera, gegen welche Frankreich und Rußland 1826 Kordons und Quarantänen errichteten, sie somit damals als kontagiös betrachteten, trat in diesen Ländern 1831 epidemisch auf, so daß ab 1832 sowohl in Großbritannien wie in Frankreich die Antikontagionisten obenausschwangen. Allgemein galten bestimmte Häuser als miasmatisch verseucht, wobei es oft dieselben waren, in denen vorher schon Tpyhusfälle beobachtet wurden ${ }^{86}$. Erst ab 1852, mit der dritten und vierten Pandemie, gewann die Kontagion wieder an Boden. Gosse, der als erster Genfer über Cholera schreibt ${ }^{87}$, betrachtet sie 1831 noch durchaus als kontagiös und vergleicht sie in dieser Hinsicht mit der Pest; die Bekämpfung soll in Quarantänen, Absonderung der Kranken, häufiger Erneuerung der verbrauchten Luft, Vorhalten eines Schwammes vor die Nase durch die Gesunden sowie Sauberkeit (häufiges Händewaschen!) und letzten Endes im Niederbrennen von "Cholerahäusern» bestehen - alles in allem ein sonderbares, aber für jene Zeit doch recht wirksames Gemisch von Maßnahmen. 1832 fügt er noch Ratschläge betreffend die diätetische Prophylaxe und Therapie der Krankheit hinzu ${ }^{88} .1833$ folgt sein großer Bericht über die Choleraepidemie

82 l.c., S. 70/71.

83 Nach E.H.Ackerknecht, s. Anmerkung 75, S. 583.

${ }^{84}$ L.-A. Gosse, Des maladies rhumatoides, Paris 1826, S. XIII.

85 l.c., S. 323.

86 Nach E.H.AcKerKnecht, l.c., S. 579.

87 L.-A. Gosse, Dissertation sur le choléra-morbus, Bibliothèque Universelle 16 (Tome 46) (1831) 188-213. Gosse weist hier u.a. auf seine eigenen Erfahrungen in Griechenland im Jahre 1828 hin (S.190).

88 L.-A. Gosse, Préservatifs et traitement du choléra, Genève 1832. 
von 1831/32 in Deutschland, Polen, Rußland und Österreich, die er im Auftrag der Helvetischen Tagsatzung zuhanden der eidgenössischen Sanitätskommission beobachtete ${ }^{89}$. Hier wird er nun deutlich Antikontagionist, indem er betont, daß die Armen zuerst von der Seuche ergriffen werden und die Hauptursache im Schmutz liegt; er beschreibt auch einzelne Cholerahäuser und gibt bei einem davon an, daß seine Bewohner aus einem Ziehbrunnen trinken, der durch das Schmutzwasser eines nahen Kanals verdreckt wird ${ }^{90} .1835$ bestätigt er diese Auffassung, indem er Kordons und Quarantänen für überflüssig erklärt und hauptsächlich die Zerstörung der Epidemieherde (Verbrennen der Häuser) empfiehlt ${ }^{91} 1842$ nimmt Gosse dann noch einmal allgemein zur Frage der Quarantänen Stellung, wobei er nun doch wieder eher zum Kontagionismus neigt und sie allgemein empfiehlt (nachdem 1841 die Pestquarantäne in England und Österreich abgeschafft worden war!), ihre Dauer aber je nach der zu bekämpfenden Seuche variiert wissen will ${ }^{92}$. LoMBards Beiträge zur Cholera beschränken sich auf eine historisch-geographische Übersicht ihres Fortschreitens von 1817 bis $1831^{93}$ und auf einen Artikel über ihre Kontagiosität aus dem Jahre 1831, in dem er sich (mangels eigener Erfahrung) der Auffassung des Pariser Chirurgen Velpeau anschließt, der zusammen mit seinem Kollegen DelPech, den Psychiatern Foville und Parchappe sowie Pariset und Moreau DE JoNNÈs die französischen Kontagionisten anführt; aus seiner Literaturübersicht zieht Lombard die in der Retrospektive recht vernünftigen Schlüsse, daß gut organisierte Sanitätskordons doch von Nutzen seien und daß beim Kontakt mit Cholerakranken Vorsicht nichts schaden könne ${ }^{94}$.

D'Espines Schriften über Cholera sind von 1835 bis 1855 rein geographische Betrachtungen, in denen er gewissermaßen mathematisch zu be-

${ }^{99}$ L.-A.Gosse, Rapport sur l'épidémie de choléra, en Prusse, en Russie et en Pologne, Genève 1833. Auszüge davon vorabgedruckt im Bulletin der eidgenössischen Sanitätskommission 1831, Nrn. 7, 8, und 1832, 10.

90 l.c., S. 116/117.

${ }^{91}$ L.-A. Gosse, Indications préservatrices et curatives pour l'épidémie de choléra, Lyon 1835, S. 30 .

92 L.-A.Gosse, De la réforme des quarantaines, mémoire adressé à Sa Majesté CharlesAlbert, Roi de Sardaigne, Genève 1842.

93 H.-C. Lombard, Notes historiques sur le choléra-morbus, Genève 1832.

94 H.-C.Lombard, Memoria sul colera-morbus di Parigi, dei dottori Trompeo e de Rolandis (Besprechung durch LOMBARD, der auch seine eigene Meinung zufügt), Bibliothèque Universelle 17 (Tome 51) (1832) 54-67, besonders 67. 
weisen versucht, daß die Schweiz verschont bleiben werde; die Choleraepidemie von Genf im Jahre 1855, deren erste zwei Fälle ausgerechnet in dem von D'EspINe ärztlich betreuten Gefängnis auftraten, belehrte ihn eines Besseren. D'Espines Optimismus hinsichtlich der Schweiz stützt sich auf die Beobachtung, daß die Cholera bis dahin «elliptisch» fortschritt und Berggebiete verschonte ${ }^{95}$, woraus er auf eine «repulsive Kraft der Alpen » schließt ${ }^{96}$. Die Genfer Epidemie von 1855, die mit 92 konstatierten Fällen (wovon 50 starben) bei einer Stadtbevölkerung von 38000 glimpflich ablief, verfolgt er sehr genau mittels an die Ärzte gesandter Fragebogen. Dieses «field trial» gestattet ihm eine präzise Schilderung des Epidemieverlaufs in einer relativ kleinen Stadt, wo die Cholera von einem ersten Herd langsam in die nähere Umgebung dringt, dabei einzelne Häuser überspringt, und schließlich in entferntere Quartiere geschleppt wird ${ }^{97}$. Da sich aber die Erkrankung im Gefängnis, wo sie zuerst auftrat ${ }^{98}$, nicht weiterverbreitete, lehnt D'EsPINE die Kontagion $a b^{99}$, was damals schon langsam unmodern wurde, und entwickelt ein kompliziertes Ineinanderwirken von Klimafaktoren und «tension épidémique». Diese spielt für ihn auch noch 1857 die Hauptrolle ${ }^{100}$, und die Cholera verbreitet sich über Europa durch eine Verderbnis der Atmosphäre ${ }^{101}$. RiLliet erwähnt 1856 D'Espines Statistik über die Genfer Choleraepidemie sehr lobend ${ }^{102}$ und ist ebenfalls vom Fehlen der Kontagion überzeugt ${ }^{103}$, wiewohl im Genfer Spital auf der Männerabteilung bei bestmöglicher Isolierung der Cholerakranken nur ein anderer Patient an Cholera erkrankte, während auf der Frauenabteilung

95 J.-M.D'Espine, Aurons-nous le choléra, Fédéral vom 31.Juli 1835. J.-M.D'Espine, Aurons-nous le choléra à Genève? Parallèle entre les deux invasions du choléra en Europe et inductions tirées en faveur de l'influence préservatrice des alpes sur la Suisse et les contrées environnantes, Journal de Genève vom 4. Oktober 1849.

96 J.-M.D'Espine, Aurons-nous le choléra? Journal de Genève vom 13. September 1854.

97 J.-M.D'Espine, Notice statistique sur la première invasion du choléra dans le canton de Genève, Genève 1855, S. 5.

${ }^{98}$ l.c., S. 4.

99 l.c., S. 9.

100 J.-M.D'Espine, Esquisse géographique des invasions du choléra en Europe. Du rôle qu'a joué la Suisse en particulier et théorie de la propagation du choléra, Bibliothèque Universelle 34 (1857) 186-221, besonders 214/215.

101 l.c., S. 214.

102 F. Rilliet, Le choléra à Genève pendant les mois de septembre et d'octobre 1855 , Union médicale 10 (1856) 145-146, 151-152, 157-158, besonders 146.

103 l.c., S. 151. 
mit weniger gründlicher Absonderung der Cholerapatientinnen fünf andere Cholerafälle auftraten ${ }^{104}$. In der Stadt fällt ihm auf, daß das Haus mit den meisten Cholerapatienten vor 13 Jahren der Schauplatz einer Epidemie von «fièvre typhoide» (oder «dothiénentérie») war; da aber auch der Typhus damals allgemein als wenig oder nicht ansteckend galt, beweist dies für ihn nur um so eindrücklicher die Existenz der «tension épidémique», die über diesem Haus offenbar besonders groß ist ${ }^{105}$. Als Prophylaxe empfiehlt er daher den Wegzug aus solchen Häusern ${ }^{106}$.

Interessanter sind die Beiträge der Genfer Hygieniker zum FleckfieberTyphus-Problem, wenn sie auch nur erste kleine Hinweise auf die Verschiedenheit der beiden Krankheiten darstellen. Früheste Andeutungen von Unterschieden zwischen der durch Läuse übertragenen, sehr ansteckenden Rickettsiose und der peroralen, vergleichweise nicht sehr ansteckenden Schmutzinfektion finden sich schon im 17.Jahrhundert; im 18. Jahrhundert unterschied Huxнam böse putride von langsamen nervösen Fiebern. Wirkliche Fortschritte brachte erst die pathologisch-anatomische Abklärung des Unterleibstyphus durch Prost (1804), Petit und Serres (1814), Nathan Smith (1824), Pretonneau (1826) und vor allem Louis (1829); bei ihm findet sich auch schon der Hinweis darauf, daß bei etlichen «Typhus»-Fällen die typischen Läsionen der Peyerschen Plaques nicht nachgewiesen werden konnten ${ }^{107}$. Die Differenzierung zwischen «englischem» Typhus (Fleckfieber) und «französischem» Typhus (Unterleibstyphus) aber ist ein Werk der amerikanischen LouIs-Schüler, wobei D'EsPINE und LoMbard mit wichtigen Hinweisen vorangingen. Leider hat D'Espine seine diesbezüglichen Beobachtungen aus dem Jahre 1833 nicht veröffentlicht, sondern erst $1853 \mathrm{kurz}$ darauf hingewiesen. In seiner «Note sur deux Typhus qui régnèrent à quelques années d'intervalle dans le bagne de Toulon. Rédigée en $1833{ }^{108}$ beschreibt er zwei Epidemien im Bagno

104 l.c., S. 151.

105 l.c., S. 151.

106 l.c., S. 157.

107 P.-Сн.-A.Lours, Recherches anatomiques, pathologiques et thérapeutiques sur la maladie connue sous les noms de gastro-entérite, fièvre putride... typhoide etc., Paris 1829, Band 2, S. 410: «... observations dans lesquelles la plupart des symptômes de l'affection typhoide ont eu lieu, sans altération spéciale des plaques elliptiques de l'iléum.»

108 J.-M.D'Espine, Parallèle entre le typhus et l'affection typhoide. A propos d'une notice inédite rédigée en 1833, sur le typhus dans le bagne de Toulon, Recueil des travaux de la Société Médicale de Genève 1 (1853) 119-141, besonders 120. 
von Toulon (1829 und 1833), von deren erster ihm der Gefängnisarzt mündlich berichtete und deren zweite er während einiger Tage selber erforschte. 1829 erkrankten von 3000 bis 4000 Sträflingen innert kürzester Zeit 1050, wovon 150 starben. Die Symptome waren Stupor, Verwirrung, fauliger Belag der Mundhöhle und der Zunge, selten Durchfall. Pathologische Veränderungen wurden kaum gefunden, insbesondere erschienen die Peyerschen Plaques gesund. Die zweite Epidemie hatte bei D'Espines Besuch seit vierzehn Tagen 110 Sträflinge erfaßt, von denen bereits 8 gestorben und noch keiner wieder gesundet war. Als Symptome imponierten Fieber, Kopfschmerz, Stupor und allgemein Verlangsamung, trockene belegte Zunge; es traten kein epigastischer Schmerz, kein Meteorismus, kein Durchfall, kein Erbrechen und keine Typhusroseolen, dafür mehr oder weniger uncharakteristische Flecken auf der Haut auf. Die Ärzte des Bagnos nannten die Krankheit «gastro-encéphalite» oder «broncho-encéphalite ataxique». Sieben früher ausgeführte Autopsien hatten eine starke Injektion der Meningen und einen normalen Verdauungstrakt ergeben; bei zwei Leicheneröffnungen, denen D'EsPINE beiwohnte, zeigte sich eine starke Injektion des Hirns und der Meningen sowie neuerdings keine veränderten Peyerschen Plaques. D'Espine schließt daraus, daß hier offbenbar etwas nach Symptomen und pathologischer Anatomie vom Typhus Verschiedenes vorliegen muß. Da erst Louis 1829 definitiv die diversen «fièvres continues» von Pinel unter dem Namen "fièvre typhoide» vereinigt und dessen Charakteristika hervorgehoben hatte, konnte D'EsPINE 1853 mit Recht von seinen 1833 verfaßten Notizen sagen: «Le typhus du bagne de Toulon est donc la première épidémie de typhus qui ait pu être observée depuis qu'on connaît bien l'affection typhoide ». ${ }^{109} \mathrm{~W}$ as mag ihn veranlaßt haben, die Notiz nicht 1833 zu veröffentlichen? Möglicherweise erschien sie dem anspruchsvollen Louis-Schüler zu fragmentarisch. Zweifellos handelte es sich hier um Fleckfieberepidemien.

Über den Typhus publizierte D'Espine nur einmal, nämlich 1849 eine Übersicht über die 357 Typhustodesfälle in Genf von 1838 bis $1845^{110}$. Auf Grund seiner sehr sorgfältigen Studie kommt er zum Schluß, daß der Typhus "plus contagieux qu'épidémique» sei, was zweifellos für die damaligen Verhältnisse stimmt: der Typhus war endemisch und ansteckend.

109 l.c., S. 125.

110 J.-M.D'Espine, Notice étiologique sur l'affection typhoide, Archives générales de médecine, 19 (1849) 129-156, 428-453. 
Im allgemeinen galt damals die Krankheit noch (im Gegensatz zum Fleckfieber, das aber bis 1850 nur selten davon abgetrennt wurde) als nicht kontagiös; für die Kontagion hatten sich einzig Leuret, Bretonneau, GeNDRON und in vorsichtiger Weise auch LouIs ausgesprochen.

Lombards Beitrag zum Typhus-Fleckfieber-Problem stammt aus dem Jahre 1836 und ist die Frucht einer Englandreise; dort fiel dem eingefleischten Louis-Schüler mit seiner großen Erfahrung auf dem Typhusgebiet ${ }^{111}$ erst einmal das Fehlen von Veränderungen der Peyerschen Plaques beim «englischen» Typhus auf; ferner zeigte dieser keine echten Roseolen, sondern Petechien, war kaum mit Durchfall oder überhaupt abdominalen Erscheinungen verbunden und schien eine viel größere Kontagiosität an den Tag zu legen als der (nach LoMBARD ebenfalls, aber in viel geringerem Ausmaß) kontagiöse «französische» Typhus ${ }^{112}$. Nach seinem Besuch der Spitäler von Dublin und Glasgow kann sich LoMBaRd noch nicht entschließen, den «englischen» Typhus als wirklich vom «französischen» verschieden zu betrachten. Eine Visite der Fieberspitäler in Manchester, Birmingham und London zeigt ihm aber neuerdings das Überwiegen der Hirn- über die Abdominalsymptome, die besondere Art der Hauteruptionen, die Abwesenheit der Diarrhoe, die Häufigkeit von Hirn- und die Seltenheit von Abdominalkomplikationen (keine Ulceration der PeYerschen Plaques), worauf er zum Schluß kommt: "Your fever is what the French pathologists have called 'typhus, typhus contagieux, fièvre des armées, fièvre des prisons'; as it can be found from the delineations written in 1813,14 and 15, when this fever prevailed wherever the armies met or sojourned... I consider Ireland as the source of the continued typhous fever which prevails in Great Britain; it is there that it is generated, or rather constantly transmitted from one individual to another." 113 Aber auch der «französische» Typhus existiert in England. Lombard faßt folgendermaßen zusammen: es gibt (jedenfalls in England) den «irischen» Typhus, welcher dem «Armeefieber» der Franzosen entspricht, sehr kontagiös ist und hauptsächlich Hirnsymptome zeigt; und einen gewöhnlichen Typhus, entsprechend dem «französischen», der sporadisch auftaucht, nicht so infektiös ist und bei dem Abdominalsymptome sowie Darmulcerationen vorherrschen.

111 H.-C.Lombard, Observations Suggested by a Comparison of the Postmortem Appearances Produced by Typhous Fever in Dublin, Paris and Geneva, Dublin 1836, S. 5.

112 l.c., S. 7.

113 H.-C.Lombard, Observations on Typhous Fever, Dublin 1836, S. 5. 
Die Pariser Académie de Médecine setzte im selben Jahr einen Preis aus für die beste Arbeit über die Analogien und Unterschiede zwischen Typhus und Fleckfieber. Gaultier de Claubry betrachtete die beiden Krankheiten als identisch, Montault kam zu keinen sicheren Schlüssen ${ }^{114}$. Dem amerikanischen Louis-Schüler Gerhard (und seinem Mitarbeiter Pennock) gelang dann anläßlich einer Fleckfieberpeidemie in Philadelphia im Jahre 1836 eine erste auf ausreichende pathologisch-anatomische Unterlagen aufgebauten Unterscheidung der beiden Affektionen ${ }^{115}$. Derselben Meinung waren 1837 Staberoh, 1839 Valleix und Shattuck sowie 1840 Rochoux und Stewart, wobei aber die Differentialdiagnose erst nach den Untersuchungen von William Jenner (1849) und Murchison (1862) sich allgemein durchsetzte.

1839 spricht sich LOMBARD auf Grund seiner eigenen Beobachtungen als Chefarzt des Genfer Spitals für die (damals noch allgemein verneinte oder für gering gehaltene) Kontagiosität des Typhus aus; ferner prüft er den Einfluß der Jahreszeiten auf die Erkrankungsziffern und findet vermehrt Typhusfälle im Sommer und Herbst ${ }^{116}$. Dieselben Thesen hält er auf Grund seiner erweiterten Erfahrung 1843 aufrecht ${ }^{117}$.

Rilliet beschäftigte sich in seiner Dissertation 1840 mit dem bis dahin noch wenig erforschten Typhus der Kinder, der damals als sehr selten galt. In einer sorgfältigen Studie im Stile von Louis und auf Grund von 16 Autopsien beweist Rilliet das recht häufige Vorkommen dieser Krankheit bei Kindern. In Anschluß an die Pariser Lehrmeinung lehnt er die Kontagion allgemein ab, kann sich aber in drei Fällen als guter Beobachter des Eindrucks nicht erwehren, daß die Kinder von ihren vorher erkrankten Eltern angesteckt wurden ${ }^{118}$.

Abgesehen von den Pionierleistungen D'Espines und Lombards hinsichtlich der Abgrenzung des Fleckfiebers vom Typhus, die zwar nicht mit Louis' numerischer Methode zustande kamen, aber doch reine Krankheitsbeschreibungen im Stile des Lehrmeisters mit Hauptakzenten auf der Symptomatologie und der pathologischen Anatomie darstellen, ist auch

114 Siehe J.-M.D'Espine (Anmerkung 108), S. 125.

115 W.W.Gerhard, On the Typhus Fever, Amer.J.Med.Sci.19 (1837) 302-303.

116 H.-C.Lombard, Etudes cliniques sur divers points de l'histoire des fièvres bilieuses et typhoides, Gazette médicale de Paris 7 (1839) 134-143, besonders 138/139.

117. H.-C.Lombard, Etudes cliniques sur quelques points de l'histoire des fièvres typhoides, Gazette médicale de Paris 11 (1843) 591-595, 606-611, 621-628, 639-643.

118 F. Rilliet, De la fièvre typhoide chez les enfants, Paris 1840, S. 78. 
bei der Seuchenhygiene - wie bei der Klimahygiene - die Zuwendung zu mehr praktischen Problemen (Beginn und Verlauf der Epidemien, die mittels Fragebogen in einer Art «field trial» verfolgt werden; Ätiologie, d.h. vor allem die Frage Kontagion oder nicht; Prophylaxe und Therapie) für die Genfer Hygieniker typisch. Sie standen dabei als Kleinstadtärzte mit ihren besonderen Erfahrungen meist im Gegensatz zu der von der Pariser Schule vertretenen antikontagionistischen Grundanschauung ${ }^{119}$, womit sie letzten Endes recht behalten sollten. Zu einer klaren Konzeption ihres Kontagionismus gelangten sie auf Grund ihrer doch nur spärlichen Ergebnisse allerdings ebensowenig wie begünstigtere Kollegen, die ausgedehntere Epidemien untersuchen konnten.

\section{3. «Humanitäre» Hygiene}

Auf diesem etwas heterogenen Gebiet, welches hier vor allem die Verbesserung der Zustände in den Gefängnissen, die Alkoholfrage sowie Probleme der menschlichen Wohnung umfaßt, ging in Genf Gosse mit seinen 1837 beginnenden Untersuchungen über das Gefängniswesen voran ${ }^{120}$, die er unermüdlich fortsetzte und in weiteren Publikationen $1840^{121}, 1863^{122}$ sowie $1864^{123}$ zur Kenntnis brachte. Er forderte vor allem eine praktische Bauweise, die eine allzu scharfe Überwachung und zu drastische Disziplin überflüssig machen sollte, sowie Erziehung der Gefangenen zur Arbeit und Wiedereingliederung in die Gesellschaft - Ziele, die seit Howard immer mehr in den Vordergrund traten, von deren Verwirklichung die Schweiz

119 Siehe z.B. bei Lombard (Anmerkung 116), S. 143: «J'ai souvent observé que la fièvre typhoide se transmettait d'individu à individu, et je fais connaître dans le mémoire quelques-unes des faits qui ont entraîné ma conviction sur la nature contagieuse de cette maladie. Cette conviction se trouve en harmonie avec celle que la plupart des médecins de province ont tirée de leurs observations; tandis que, dans la capitale, les principaux praticiens affirment n'avoir rien vu qui pût les conduire à considérer la fièvre typhoide comme contagieuse; mais il faut se rappeler qu'en matière d'observation, des faits négatifs, quelque nombreux qu'ils soient, ne peuvent détruire l'évidence déduite de faits positifs et bien avérés. »

120 L.-A. Gosse, Examen médical et philosophique du système pénitentiaire, Genève 1837.

121 L.-A. Gosse, Examen du projet de loi sur les prisons et du plan de la nouvelle maison de détention de Genève, Genève 1840.

122 L.-A. Gosse. Enquête pénitentiaire Suisse. Canton de Genève, Genève 1863. L.-A. GossE, Enquête sur les prisons de la Suisse, Genève 1863.

123 L.-A. Gosse, Rapport sur la question pénitentiaire en Suisse, Zurich 1864. L.-A. GossE, Projet d'un pénitentier industriel, agricole et éducatif etc., Genève 1864. 
damals aber nocht weit entfernt war. Der Gefängnisarzt D'Espine unterstützte diese Bemühungen von Gosse durch Veröffentlichungen über die den Gefangenen zustehende minimale Raumgröße (1837) ${ }^{124}$ sowie das Verhalten des Körpergewichts der Sträflinge nach ihrer Einlieferung (1844): allgemein nahmen die Gefangenen in Genf leicht ab, wobei diese Abmagerung bei den am strengsten Gehaltenen am deutlichsten hervortrat ${ }^{125}$. Zum Alkoholismus äußert sich u. a. Lombard 1841, der den Alkohol für eine lange Reihe von Krankheiten verantwortlich macht und die Bekämpfung dieses Übels fordert ${ }^{126}$. Antoine Baumgartner (1808-1895), ebenfalls Louis-Schüler, aber mehr als Politiker denn als Arzt tätig, Autor zahlreicher Zeitungsartikel über praktische hygienische Fragen ${ }^{127}$, versucht gleichzeitig darzutun, daß der Alkoholismus in Genf nicht sehr verbreitet ist $^{128}$. Gosse greift die Frage 1857 erneut auf ${ }^{129}$, schließt sich dabei den Ansichten von Lombard an und versucht dem Problem durch die Propagation anderer billiger Getränke beizukommen ${ }^{130}$. Zur selben Zeit macht er Vorschläge für die Verbesserung der hygienischen Verhältnisse in ländlichen Wohnungen ${ }^{131}$, während D'Espine schon vorher über das Trocknenlassen

124 J.-M.D'Espine, Rapport à la Commission administrative des prisons sur un projet de diminuer l'espace des cellules, Genève 1837.

125 J.-M.D'Espine, Notice sur les variations du poids des prisonniers soumis au régime pénitentiaire, Annales d'Hygiène Publique et de Médecine Légale 32 (1844) 71-85, besonders 78.

${ }^{126}$ H.-C. Lombard, Lettre du $D^{\mathrm{r}}$ Lombard au $D^{\mathrm{r}}$ Coindet touchant l'influence de l'ivrognerie sur la production d'un grand nombre de maladies, Genève 1841.

127 u. a. 1853: Agrandissement de la ville. Opération des fortifications. 1854: Logement des ouvriers. 1855: Asile des vieillards. 1858: Effets généraux des chemins de fer. 1860: Garde médicale de la Ville de Genève. 1864: Questions sanitaires. Des moyens d'assainir l'abattoir. Documents sur une industrie insalubre. Hygiène publique. 1868: Percement des rues. 1873: Persépolis ou essai sur l'amélioration de la Ville de Genève (zum Teil zitiert nach A.-J.Baumgartner, Le Docteur Baumgartner. Souvenirs de sa vie et de ses écrits, Genève 1895, S. 93).

128 A.Baumgartner, Contre-enquête sur la question de l'ivrognerie ou justification du peuple de Genève, Genève 184.1.

129 A.-A. Gosse, Mémoire sur les moyens de prévenir et combattre l'ivrognerie. Congrès international de bienfaisance de Bruxelles 1857.

130 L.-A.Gosse, Des boissons fermentées économiques, Genève 1857. L.-A.Gosse, Rapport sur la fabrique d'eau minérales de M. Louis Gerbel, Bulletin de la Société des Arts de Genève 1859, $\mathrm{n}^{\circ}$ 68, p. 1-19.

131 L.-A.Gosse, Des habitations rurales. Principales conditions hygiéniques et économiques à observer dans leur constructions, Bulletin de la Société Genevoise d'Utilité Publique 1 (1858) 65-100. 
neuerbauter Häuser publiziert hatte ${ }^{132}$. Zum Sammelkapitel «humanitäre Hygiene» können schließlich auch noch die Artikel von Gosse, Prévost, DupiN und LombarD über das «Dispensaire» gezählt werden, worin sie über den Nutzen und das gute Funktionieren dieser frühen kostenlosen Poliklinik berichten ${ }^{133}$.

\section{Gewerbehygiene}

Einige vielversprechende Anfänge auf diesem Gebiet in der damals etwa im Gegensatz zu England oder Frankreich - noch sehr wenig industrialisierten Schweiz des 18. Jahrhunderts stammen von den Genfern P.-F. Tingry (1743-1821) ${ }^{134}$ und H.-A.Gosse (1753-1816, Vater von L.-A. $\operatorname{Gosse}^{135}$, die sich mit den Berufskrankheiten der Metallvergolder und der Arbeiter in Hutfabriken befaßten. LouIs-André Gosse wollte das Werk seines Vaters fortsetzen und entwarf in seiner Dissertation eine kurze theoretische Übersicht über die vordringlichsten Fragen, die er offensichtlich später zu untersuchen im Sinne hatte ${ }^{136}$. Nach seiner Rückkehr in die Heimatstadt gelang ihm aber nur noch eine einzige kleine Arbeit in dieser Richtung, welche sich mit den allgemeinen Schutzmaßnahmen bei ungesunden Arbeitsverhältnissen befaßt. Gosse führte dabei auch Selbstversuche durch und empfahl allgemein bei staubigen oder mit Emanationen verschiedener Stoffe verbundenen Arbeiten das Vorbinden eines nassen Schwamms vor die Nase ${ }^{137}$. Etwas tiefer drang sein Kollege Lombard in das Gebiet ein, indem er den Einfluß der Berufsarten auf die Lungentuberkulose sowie auf die Lebensdauer studierte. Er untersuchte u.a. 8829

132 J.-M.D'Espine, Moyen de juger jusqu'à quel point une maison récemment bâtie est assez sèche pour être habitée impunément, Annales d'Hygiène êt de Médecine Légale 3 (1855) 291-301.

133 L.-A. Gosse, J.-L.Prévost und J.-P.Dupin, Rapports du Dispensaire de Genève, $1^{\mathrm{re}}$ année, Genève 1823. L.-A.Gosse, J.-L.PrÉvost und J.-P.Dupin, Second rapport du Dispensaire de Genève, Genève 1824. H.-C.Lombard, Troisième rapport du Dispensaire de Genève (zusammen mit den Obgenannten), Genève 1830.

134 P.-F.Tingry, Mémoire sur les moyens de préserver les doreurs en pièces de montres des pernicieux effets du mercure en vapeurs, Genève 1777.

135 H.-A.Gosse, Mémoire sur la question suivante: Déterminer les maladies auxquelles sont exposés les doreurs sur métaux et la meilleure manière de les en préserver, Genève 1783.

136 L.-A. Gosse, Propositions générales sur les maladies causées par l'exercice des professions, Paris 1816.

137 L.-A.Gosse, Sur l'hygiène des professions insalubres. De l'emploi des masques et des lunettes d'éponges, Bibliothèque Universelle 2, Tome 4 (1817) 57-69. 
Todesfälle in Genf, von denen 1003 durch ein Phthise bedingt waren, und arbeitete die am meisten gefährdeten Berufe sowie die Art der vermutlichen Noxe heraus. Nach seinen Forschungen fördert besonders der mineralische Staub die Phthise (bei Steinmetzen, Bildhauern usf. fand er 177 Phthisiker auf 1000 Todesfälle), während der vegetabilische Staub (Müller, Bäcker) und der animalische (Hut-, Pelz- und Matrazenmacher) in dieser Hinsicht weniger gefährlich sind (105 und 144. Phthisiker auf 1000 Todesfälle). Sein Pariser Kollege Benoiston de Chateauneuf fand dagegen den animalischen Staub an erster Stelle, gefolgt vom pflanzlichen und mineralischen. Da zu jener Zeit die Anteile der Pneumonokoniose, der Silikose und der Tuberkulose am Lungenschaden nicht ausreichend genau bestimmt werden konnten, scheint LoMBards Ansicht eher überzeugender, wiewohl ihm in Genf keine Kohlengruben- und sonstigen Bergwerksarbeiter zur Verfügung standen, die das Bild wohl noch mehr zugunsten seiner Meinung verschoben hätten ${ }^{138}$. Als Prophylaxe und Therapie empfiehlt er den besonders gefährdeten Arbeitern im Falle eines Verdachts auf Tuberkulose oder einer festgestellten Phthise den Aufenthalt in frischer Luft sowie einen entsprechenden Berufswechsel. Tuberkulosegefährdete Kinder sollten von Anfang an wenn möglich leichtere und in frischer Luft ausübbare Berufe ergreifen können ${ }^{139}$. Die Zusammenhänge zwischen Beruf und Lebensdauer untersuchte LoMbard anhand von 8488 männlichen Verstorbenen (Eintragungen im Genfer «Registre mortuaire» von 1796 bis 1830), deren mittlere Lebensdauer $55 \mathrm{Jahre}$ betrug. Über diesem Mittel kommen u. a. die Ärzte zu liegen. Als günstige Faktoren für die Lebensdauer eruiert Lombard das (finanzielle) Wohlergehen sowie eine aktive Lebensform. Ungünstig sind die Misere, Dämpfe verschiedener Art sowie Staub, besondere Exposition (Dachdecker, Kutscher, Metzger), ferner - schon damals - eine sitzende Lebensweise ${ }^{140}$. Etwa zur selben Zeit beschreibt sein Kollege Rilliet in Paris einen Fall von Melanose der Lungen, wobei er zum Schluß kommt, daß die Einatmung von Kohlenstaub nicht die Phthise erzeugt, aber den Zustand der Tuberkulose verschlimmern kann ${ }^{141}$.

138 H.-C.Lombard, De l'influence des professions sur la phthisie pulmonaire, Annales d’Hygiène Publique et de Médecine Légale 11 (1834) 5-76, besonders 57/58.

139 l.c., S. 69.

140 H. C.Lombard, De l'influence des professions sur la durée de la vie. Recherches statistiques, Mémoires de la Société de Physique et d'Histoire naturelle de Genève 7 (1836) 77-120, besonders 112.

141 F.Rilliet, Mémoire sur la pseudo-mélanose des poumons, Archives générales de médecine 2 (1838) 160-174. 
Rechtmäßiger Erbe der reichen Tradition, welche die OdIER, D'Ivernois, Gosse, D'Espine, Lombard, Baumgartner und andere mit ihren gewissermaßen privaten Studien über Medizinalstatistik und Hygiene verschiedener Bezirke des menschlichen Lebens begründet hatten, wurde im Jahre 1876 Pierre-Louis Dunant (1834-1918), ein jüngerer Bruder des Rotkreuz-Stifters Henri Dunant (1828-1910), indem er die Professur für Hygiene an der neugegründeten Genfer Universität einnahm, die er bis 1889 innehielt. Er hatte noch vor seiner Promotion in Paris (1863) vor allem mit D'EspINe zusammengearbeitet und setzte die alte Überlieferung in vielen Veröffentlichungen würdig fort. Auch er weist den für jene Genfer Epoche typischen humanitären Zug auf: er unterstzützte seinen Bruder Henri bei der Entwicklung des Rotkreuzgedankens und gründete 1870 das "Asyle de Pressy» für bedürftige Kranke, in welchem er selber vierzig Jahre lang wirkte. Mit ihm beginnt in Genf die Epoche der akademischen Forschung in Hygiene und Medizinalstatistik, welche vor allem durch die hier besprochenen Werke von Gosse, d'Espine und LoMbard eine gründliche Vorbereitung erfahren hatte. 